DRAFT V2

Preprint typeset using $\mathrm{LATEX}_{\mathrm{E}}$ style emulateapj v. 12/16/11

\title{
BIMODAL LONG-LASTING COMPONENTS IN SHORT GAMMA-RAY BURSTS: PROMISING ELECTROMAGNETIC COUNTERPARTS TO NEUTRON STAR BINARY MERGERS
}

\author{
Shota Kisaka $^{1,3}$, Kunihito IokA ${ }^{2}$, And TAKanori Sakamoto ${ }^{1}$ \\ draft v2
}

\begin{abstract}
Long-lasting emission of short gamma-ray bursts (GRBs) is crucial to reveal the physical origin of the central engine as well as to detect electromagnetic (EM) counterparts to gravitational waves (GWs) from neutron star binary mergers. We investigate $65 \mathrm{X}$-ray light curves of short GRBs, which is six times more than previous studies, by combining both Swift/BAT and XRT data. The light curves are found to consist of two distinct components at $>5 \sigma$ with bimodal distributions of luminosity and duration, i.e., extended (with timescale $\lesssim 10^{3} \mathrm{~s}$ ) and plateau emission (with timescale $\gtrsim 10^{3}$ s), which are likely the central engine activities but not afterglows. The extended emission has an isotropic energy comparable to the prompt emission, while the plateau emission has $\sim 0.01-1$ times of that energy. A half (50\%) of our sample has both components, while the other half is consistent with having both components. This leads us to conjecture that almost all short GRBs have both the extended and plateau emission. The long-lasting emission can be explained by the jets from black holes with fallback ejecta, and could power macronovae (or kilonovae) like GRB 130603B and GRB 160821B. Based on the observed properties, we quantify the detectability of EM counterparts to GWs, including the plateau emission scattered to the off-axis angle, with $C A L E T / \mathrm{HXM}, I N T E G R A L / \mathrm{SPI}-$ ACS, Fermi/GBM, MAXI/GSC, Swift/BAT, XRT, future ISS-Lobster/WFI, Einstein Probe/WXT, and $e R O S I T A$.
\end{abstract}

Subject headings:

\section{INTRODUCTION}

The physical origin of short gamma-ray bursts (GRBs) remains unknown despite intensive studies (e.g., Nakar 2007; Lee \& Ramirez-Ruiz 2007). The leading model for a progenitor of short GRBs is neutron star (NS) binary mergers including NS-NS and black hole (BH)-NS binaries (e.g., Naravan et al. 1992), as inferred from the short emission timescale, the energetics, and the wide variety of their host galaxy type (Berger 2014). However, there is no smoking-gun evidence for the merger hypothesis yet.

The best way to verify the merger hypothesis is to detect coincident gravitational waves (GWs). Recently, the GW astronomy has begun since the direct detections of GWs from BH-BH binaries, GW 150914, LVT 151012, GW 151226, and GW 170104 by Advanced LIGO (Abbott et al. 2016a, b, c, 2017). An NS binary merger is also associated with the GW emission that is detectable by the current GW detectors (Abbott et al. 2016d). Simultaneous detection of GW and electromagnetic (EM) emission, particularly the association to short GRBs, will provide valuable information for understanding the progenitor of short GRBs (e.g., Metzger \& Berger 2012; Fan et al. 2017).

Activities of the central engine of short GRBs continue much longer timescale $(\gg 1 \mathrm{~s})$ than the duration of the prompt emission ( $\lesssim 1$ s) (e.g., Barthelmy et al. 2005;

\footnotetext{
kisaka@phys.aoyama.ac.jp

kunihito.ioka@yukawa.kyoto-u.ac.jp

tsakamoto@phys.aoyama.ac.jp

${ }^{1}$ Department of Physics and Mathematics, Aoyama Gakuin

University, Sagamihara, Kanagawa, 252-5258, Japan

2 Center for Gravitational Physics, Yukawa Institute for Theoretical Physics, Kyoto University, Kyoto 606-8502, Japan

3 JSPS Research Fellow
}

Ioka et al. 2005; Norris \& Bonnell 2006). Swift/BAT has detected more than 100 short GRBs, and $\sim 70 \%$ of them have been also detected by Swift/XRT 4 . Some Swift/XRT light curves show a long duration with rapid flux decline, which is only produced by activities of the central engine (e.g., Ioka et al. 2005). By investigating the properties of the long-lasting components, we can obtain a clue to the central engine of short GRBs, and improve the strategies for the simultaneous detection with GW emission.

One of the long-lasting activities in short GRBs is the extended emission with timescale $\sim 100 \mathrm{~s}$, which is much longer than typical accretion timescale (e.g., Barthelmy et al. 2005; Norris \& Bonnell 2006). In some bursts, the fluence of the extended emission is comparable to or even higher than that of the initial pulse (e.g., Perley et al. 2009). The fraction of bursts with the extended emission in the $\gamma$-ray band $(>15 \mathrm{keV})$ is $\sim 2-25 \%$ (e.g., Norris et al. 2010 ; Sakamoto et al. 2011; Bostanci et al. 2013; Kaneko et al. 2015; Lien et al. 2016). However, as shown later, a larger number of the extended emission component may be missed in the Swift/BAT band (15-150 keV), because some bursts show extended emission only in the Swift/XRT band (0.3$10 \mathrm{keV}$; Rowlinson et al. 2013; Kagawa et al. 2015; Lü et al. 2015, 2017).

In addition, short GRBs with extended emission in the $\gamma$-ray band also have plateau emission with timescale $\sim 10^{3}-10^{4} \mathrm{~s}$ in the Swift/XRT band (Gompertz et al. 2013, 2014). Some theoretical models for the plateau emission suggest an activity of the central engine such as a relativistic jet from a $\mathrm{BH}$ with a typical NS magnetic field $\sim 10^{12} \mathrm{G}$ (Kisaka \& Ioka 2015) or a pulsar

\footnotetext{
${ }^{4}$ https://swift.gsfc.nasa.gov/archive/grb_table/
} 
wind from a highly magnetized $\left(\sim 10^{15}-10^{16} \mathrm{G}\right)$ and rapidly rotating $(\sim 1 \mathrm{~ms})$ NS (e.g., Gompertz et al. 2014; Gibson et al. 2017). Note that some of bursts without extended emission in the $\gamma$-ray band also show the plateau-like emission component (Rowlinson et al. 2013; Lü et al. 2015, 2017).

In order to increase the detectability of EM counterparts to GWs from NS binary mergers, it is important to understand the properties of the long-lasting components. For example, their luminosity function is necessary to estimate the integration time of the follow-up observations. Comparing the duration distribution with the required integration time will determine the maximum number of pointing observations. Therefore, it is important to investigate the statistical properties of the long-lasting components from the current observational data in order to improve the observational strategies for the EM counterparts.

The properties of the long-lasting components are also important to characterize the interaction between the jets and the merger ejecta surrounding the central engine (e.g., Metzger et al. 2008b; Bucciantini et al. 2012). In particular, nearly isotropic emission is anticipated through the interaction (e.g., Metzger \& Piro 2014; Nakamura et al. 2014; Kisaka et al. 2015a. b; Hotokezaka \& Piran 2015. Sun et al. 2017; Lazzati et al. 2016; Gottlieb et al. 2017). Although the plateau emission may arise from a collimated relativistic jet, a significant fraction of the emission could be scattered to wider solid angle by the merger ejecta during the plateau emission activity (Kisaka et al. 2015b). Then, the properties of plateau emission are necessary to estimate those of the scattered component. In addition, the extended and plateau emission activities could heat the merger ejecta, which is observed as a macronova 5 (or kilonova) in the optical and infrared bands (Kulkarni 2005; Yu, Zhang \& Gao 2013; Kisaka et al. 2015a,b). The emission could be brighter than the macronova heated by the decay of the heavy elements (e.g., Li \& Paczyńsky 1998; Kulkarni 2005), in particular $r$-process elements (e.g., Metzger et al. 2010b; Kasen et al. 2013; Tanaka \& Hotokezaka 2013), which is widely discussed. The luminosity of the enginepowered macronova is determined by the properties of the long-lasting components (Kisaka et al. 2015a,b).

Currently, the number of short GRBs with both the extended and plateau emission is only $\sim 10$, whose extended emission was detected in the Swift/BAT band (Gompertz et al. 2014; Kisaka \& Ioka 2015). The number is too small to statistically characterize the properties of the extended and plateau emission. On the other hand, some bursts without the BAT-detected extended emission actually show the features of the extended and plateau emission, that are flat flux evolution and rapid decline in the Swift/XRT band instead (Rowlinson et al. 2013; Lü et al. 2015, 2017). In fact, the fraction of the bursts with extended emission tends to be higher for softer threshold energy (Norris et al. 2010; Bostanci et al. 2013). Hence, both the Swift/BAT and XRT bands should be used to identify the extended

\footnotetext{
5 We use the term "macronova" as a thermal radiation from the merger ejecta of NS binaries, whatever the energy source is, like a supernova.
}

and plateau emission components (Kagawa et al. 2015). Then, the sample size of the extended and plateau emission components becomes large. With a large sample, we can investigate whether the extended and plateau emission is two distinct components or not, and whether all short GRBs have both components following the prompt emission or not.

In this paper, we investigate the light curves of 65 short GRBs with the sufficient Swift/XRT data to characterize the statistical properties of both extended and plateau emission. Using a phenomenological model, we extract the long-lasting components, the extended and plateau emission, from the observed light curves. In Section 2, we describe our sample and the light curve model. In Section 3, we provide the results of the obtained luminosity and duration of the extended and plateau emission, and show that the distributions are bimodal. In Section 4, we discuss the detectability of the long-lasting components as an EM counterpart to GW from NS binary mergers. In Section 5, we discuss implications for theoretical models based on the obtained properties of the long-lasting emission components. Conclusions and discussion are provided in Section 6 .

\section{SAMPLE AND MODEL}

In this paper, we refer to bursts as short GRBs if $T_{90} \leq 2$ s (Kouveliotou et al. 1993), where $T_{90}$ corresponds to the duration that contains $90 \%$ of the burst fluence measured by the Swift/BAT instrument (15-150 $\mathrm{keV})$. The short GRB data sample was taken from UK Swift Science Data Center 6 (Evans et al. 2007, 2009). We use the data observed by Swift/BAT 7 and XRT 8 to fit the light curve. Our sample consists of short GRBs with at least three detection points by Swift/XRT. We also include several bursts with $T_{90}>2 \mathrm{~s}$ in our sample, which are considered as short GRBs with extended emission detected by Swift/BAT (Gompertz et al. 2013; Lien et al. 2016). Table 1 lists the sample of 65 short GRBs between January 2005 and June 2017, which corresponds to about a half of the entire short GRBs detected by Swift/BAT. Our sample overlaps with that in the previous studies of Swift/XRT-detected short GRBs, which were discussed in the context of the NS engine model (Rowlinson et al. 2013; Gompertz et al. 2013, 2014; Lü et al. 2015). For the bursts without known redshift, we use the averaged value of the measured-redshifts in our sample $z=0.72$, which is in agreement with the values reported in other works $(<z>\sim 0.5-0.8$; Rowlinson et al. 2013; D'Avanzo et al. 2014; Berger 2014; Lü et al. 2015).

We show that after the prompt emission, the light curve consists of two components: the extended and plateau emission. In order to identify the extended and plateau emission components, we adopt a phenomenological formula with two functions of a constant and subsequent power-law decay,

$$
L_{\mathrm{iso}}(t)=L_{\mathrm{iso}, \mathrm{EX}}\left(1+\frac{t}{T_{\mathrm{EX}}}\right)^{-\alpha}
$$

\footnotetext{
6 http://www.swift.ac.uk/index.php

7 http://www.swift.ac.uk/burst_analyser/

8 http://www.swift.ac.uk/xrt_curves/
} 
TABLE 1 Short GRB Samples

\begin{tabular}{|c|c|c|c|c|}
\hline Name & Redshift & Reference & Extended emission & Plateau emission \\
\hline 050509B & 0.2249 & Prochaska et al. (2005) & & $\checkmark$ \\
\hline $050724^{*}$ & 0.257 & Berger et al. (2005) & $\checkmark$ & $\checkmark$ \\
\hline 051210 & $(0.72)$ & & $\checkmark$ & \\
\hline 051221A & 0.5464 & Soderberg et al. (2006) & $\checkmark$ & $\checkmark$ \\
\hline $051227^{*}$ & 0.8 & D'Avanzo et al. (2009) & $\checkmark$ & $\checkmark$ \\
\hline 060313 & $(0.72)$ & & $\checkmark$ & $\checkmark$ \\
\hline $060614^{*}$ & 0.1254 & Della Valle et al. (2006) & $\checkmark$ & $\checkmark$ \\
\hline 060801 & 1.1304 & Berger et al. (2007) & $\checkmark$ & \\
\hline $061006^{*}$ & 0.4377 & Berger et al. (2007) & $\checkmark$ & $\checkmark$ \\
\hline 061201 & 0.111 & Berger (2006) & $\checkmark$ & $\checkmark$ \\
\hline $061210^{*}$ & 0.4095 & Berger et al. (2007) & $\checkmark$ & $\checkmark$ \\
\hline 070714A & 1.58 & $\mathrm{a}$ & & $\checkmark$ \\
\hline 070714B* & 0.9224 & Cenko et al. (2008) & $\checkmark$ & $\checkmark$ \\
\hline 070724A & 0.4571 & Berger (2009) & $\checkmark$ & $\checkmark$ \\
\hline 070809 & 0.2187 & Perlev et al. (2008) & & $\checkmark$ \\
\hline $071227^{*}$ & 0.381 & D'Avanzo et al. (2009) & $\checkmark$ & $\checkmark$ \\
\hline $080123^{*}$ & 0.495 & Leibler \& Berger (2010) & $\checkmark$ & $\checkmark$ \\
\hline 080426 & $(0.72)$ & & & $\checkmark$ \\
\hline 080503* & $(0.72)$ & & $\checkmark$ & \\
\hline 080702A & $(0.72)$ & & $\checkmark$ & \\
\hline 080905A & 0.1218 & Rowlinson et al. (2010b) & $\checkmark$ & \\
\hline 080919 & $(0.72)$ & & $\checkmark$ & \\
\hline 081024A & $(0.72)$ & & $\checkmark$ & \\
\hline $081226 \mathrm{~A}$ & $(0.72)$ & & & $\checkmark$ \\
\hline 090426 & 2.609 & Levesque et al. (2010) & $\checkmark$ & $\checkmark$ \\
\hline 090510 & 0.903 & McBreen et al. (2010) & $\checkmark$ & $\checkmark$ \\
\hline 090515 & $(0.72)$ & & $\checkmark$ & \\
\hline 090621B & $(0.72)$ & & & $\checkmark$ \\
\hline 091109B & $(0.72)$ & & $\checkmark$ & $\checkmark$ \\
\hline 100117A & 0.915 & Fong et al. (2011) & $\checkmark$ & \\
\hline $100625 \mathrm{~A}$ & 0.452 & Fong et al. $(2013)$ & $\checkmark$ & $\checkmark$ \\
\hline $100702 \mathrm{~A}$ & $(0.72)$ & & $\checkmark$ & \\
\hline $100724 \mathrm{~A}$ & 1.288 & Thoene et al. (2010) & $\checkmark$ & $\checkmark$ \\
\hline 101219A & 0.718 & Fong et al. (2013) & $\checkmark$ & \\
\hline $110112 \mathrm{~A}$ & $(0.72)$ & & & $\checkmark$ \\
\hline $111020 \mathrm{~A}$ & $(0.72)$ & & & $\checkmark$ \\
\hline 111117A & 1.31 & Sakamoto et al. (2013) & $\checkmark$ & $\checkmark$ \\
\hline $111121 \mathrm{~A}^{*}$ & $(0.72)$ & & $\checkmark$ & $\checkmark$ \\
\hline $120305 \mathrm{~A}$ & $(0.72)$ & & $\checkmark$ & $\checkmark$ \\
\hline $120521 \mathrm{~A}$ & $(0.72)$ & & $\checkmark$ & \\
\hline $120804 \mathrm{~A}$ & 1.3 & Berger et al. (2013b) & $\checkmark$ & $\checkmark$ \\
\hline $121226 \mathrm{~A}$ & $(0.72)$ & & $\checkmark$ & $\checkmark$ \\
\hline 130603B & 0.3564 & de Ugarte Postigo et al. (2014) & & $\checkmark$ \\
\hline 130912A & $(0.72)$ & & & $\checkmark$ \\
\hline $131004 \mathrm{~A}$ & 0.717 & Chornock et al. (2013) & $\checkmark$ & $\checkmark$ \\
\hline 140129B & $(0.72)$ & & & $\checkmark$ \\
\hline 140516A & $(0.72)$ & & $\checkmark$ & $\checkmark$ \\
\hline $140903 \mathrm{~A}$ & 0.351 & Troja et al. (2016a) & & $\checkmark$ \\
\hline 140930B & $(0.72)$ & & $\checkmark$ & $\checkmark$ \\
\hline $150120 \mathrm{~A}$ & 0.460 & Chornock \& Fong (2015) & $\checkmark$ & \\
\hline $150301 \mathrm{~A}$ & $(0.72)$ & & $\checkmark$ & \\
\hline $150423 \mathrm{~A}$ & 1.394 & Malesani et al. (2015) & $\checkmark$ & \\
\hline $150424 A^{*}$ & 0.30 & Castro-Tirado et al. (2015) & $\checkmark$ & $\checkmark$ \\
\hline $150831 \mathrm{~A}$ & $(0.72)$ & & $\checkmark$ & $\checkmark$ \\
\hline $151127 \mathrm{~A}$ & $(0.72)$ & & & $\checkmark$ \\
\hline $151229 \mathrm{~A}$ & $(0.72)$ & & $\checkmark$ & $\checkmark$ \\
\hline $160408 \mathrm{~A}$ & $(0.72)$ & & $\checkmark$ & \\
\hline $160411 \mathrm{~A}$ & $(0.72)$ & & & $\checkmark$ \\
\hline $160525 \mathrm{~B}$ & $(0.72)$ & & $\checkmark$ & $\checkmark$ \\
\hline $160601 \mathrm{~A}$ & $(0.72)$ & & & $\checkmark$ \\
\hline $160624 \mathrm{~A}$ & 0.483 & Cucchiara \& Levan (2016) & $\checkmark$ & \\
\hline 160821B & 0.16 & Levan et al. (2016) & $\checkmark$ & $\checkmark$ \\
\hline $160927 \mathrm{~A}$ & $(0.72)$ & & $\checkmark$ & $\checkmark$ \\
\hline $161004 \mathrm{~A}$ & $(0.72)$ & & $\checkmark$ & $\checkmark$ \\
\hline 170127B & $(0.72)$ & & $\checkmark$ & $\checkmark$ \\
\hline
\end{tabular}




$$
+L_{\text {iso }, \mathrm{PL}}\left(1+\frac{t}{T_{\mathrm{PL}}}\right)^{-\alpha},
$$

where $L_{\text {iso,EX }}, L_{\text {iso,PL }}, T_{\mathrm{EX}}$, and $T_{\mathrm{PL}}$ are the isotropic luminosities and durations of the extended and plateau emission, respectively (see also Willingale et al. 2007). In Equation (1), the time after the Swift/BAT detection in the rest-frame is $t$, and the temporal index is $\alpha$. As a fiducial value, we use $\alpha=40 / 9$ implied by the BH engine model (see Section 5 for details; Kisaka \& Ioka 2015), where the exact value does not alter our conclusions unless much small value $\alpha \lesssim 2$ is assumed (see Section 6 for details). We define the extended emission as the emission with timescale $\lesssim 10^{3}$ s, some of which are not detected by Swift/BAT. For a longer timescale component $\left(\gtrsim 10^{3}\right.$ s), we define it as the plateau emission. We compare the phenomenological formula with the observations and obtain the model parameters $L_{\text {iso,EX }}, L_{\text {iso,PL }}, T_{\mathrm{EX}}$ and $T_{\mathrm{PL}}$ for each burst. We assume that the extended emission has to satisfy the condition $L_{\text {iso,Ex }} / L_{\text {iso,PL }} \gtrsim 10$, because a weak emission component is difficult to distinguish it from an X-ray flare. For the plateau emission, we require that there is at least one detection point whose luminosity is $>10$ times larger than that of the extended emission tail, $L_{\text {iso,EX }}\left(t / T_{\mathrm{EX}}\right)^{-\alpha}$ at $t>T_{\mathrm{EX}}$. Note that the identification of the extended and plateau emission is purely phenomenological.

\section{RESULTS}

Figure 1 shows the observational data points of all bursts in our sample. The phenomenological light curves in Equation (1) with $\alpha=40 / 9$ are also shown as solid curves. The gray dot-dashed curves in Figure 1 denote the possible components that were missed in the observations. We fit the phenomenological light curve to the observational data by eye inspection, because the light curves often have some additional complex structures such as X-ray flares. As shown in Figure 11, the twocomponent light curves in Equation (1) is consistent with all the observations. In some bursts, the decay of the extended emission is too sharp to fit the phenomenological model (e.g., GRB 050724; Barthelmy et al. 2005). We discuss this possible issue in Section 5 .

In Table 1, we list two components, the extended and plateau emission, seen in the observed light curves for each burst. The fraction of short GRBs with the extended emission in our sample detected by Swift/BAT and XRT is $49 / 65 \sim 0.75$. This is almost the same value, $26 / 32 \sim 0.81$, also for the redshift-measured bursts. The number of short GRBs with the extended emission is about a half of the total Swift/BAT-detected short GRBs ( 100 events). These indicate that the Swift/BATdetected short GRBs accompanying the extended emission is fairly common. The number of bursts with the plateau emission is 49 for all sample and 26 for redshiftknown sample, which are (accidentally) the same values as the extended emission. The number of short GRBs with both the extended and plateau emission is 33 , which is a half of our sample. Therefore, the association of both components is also common for short GRBs.

Figure 2 shows the luminosity (left panels) and duration distributions (right panels) for the Swift/BATdetected (light-blue) and BAT-non-detected extended emission (blue), and plateau emission (red). The his- tograms show a hint of a bimodal distribution. To quantify the bimodality in the histograms, we perform the Hartigan's Dip Test (Hartigan 1985) using 'diptest' CRAN package of the $\mathrm{R}$ software. The null hypothesis of this test is that a distribution is a unimodal distribution. The null probabilities of the dip test of the luminosity distributions (Figure 2 (A), (C), (E) and (G)) are $0.404,0.874,0.341$ and 0.539 for the samples of all GRBs, redshift-known GRBs, GRBs with both the extended and plateau components and GRBs with the single component, respectively. No statistically significant bimodality is evident in the luminosity distributions. However, in the duration distributions (Figure 2 (B), (D), (F) and $(\mathrm{H})$ ), the null probabilities are $3.67 \times 10^{-3}, 3.91 \times 10^{-3}$, $4.10 \times 10^{-3}$ and $1.73 \times 10^{-2}$ for the samples of all GRBs, redshift-known GRBs, GRBs with both the extended and plateau components and GRBs with the single component, respectively. Therefore, the duration distributions reject a unimodality, and prefer a bimodal distribution. Based on this Dip Test, the extended and plateau emission are very likely distinct populations. Namely, there are two distinct long-lasting components following the prompt emission in short GRBs. This is the first indication that two distinct long-lasting components are ubiquitous in short GRB light curves as far as we know (see the cluster analysis in Figure 4 for stronger evidence). A normal afterglow cannot explain the plateau-like temporal evolution in the light curve (e.g., Sari et al. 1998). The presence of the long-lasting components means that there are at least two activity phases related to the central engine in addition to the prompt emission. Our findings would not only serve as a key to the final understanding of the short GRBs, but also provide appropriate strategies to detect short GRBs as an EM counterpart to a GW source (Section 4).

Figures 2 (A) and (B) show the distributions for all bursts in our sample. From Figure 2 (A), the luminosity range of the extended emission is $10^{47} \mathrm{erg}$ $\mathrm{s}^{-1} \lesssim L_{\text {iso,EX }} \lesssim 10^{50} \mathrm{erg} \mathrm{s}^{-1}$, which is somewhat broader than that of the extended emission detected by Swift/BAT $\left(10^{48}\right.$ erg s $^{-1} \lesssim L_{\text {iso,EX }} \lesssim 10^{50}$ erg s$^{-1}$; e.g., Gompertz et al. 2013, 2014). The duration distribution of the extended emission is concentrated in $T_{\mathrm{EX}} \sim 200$ s (Figure 2 B). This narrowness of the $T_{\mathrm{Ex}}$ distribution might be the intrinsic properties, or the observational bias that we are missing the extended emission with shorter duration due to time lag between the BAT triggering time and the observational starting time of Swift/XRT, $\sim 60-100$ s. The luminosity and duration of the Swift/BAT-detected and non-detected extended emission are continuously distributed, so that both populations would be the same component. The luminosity range of the plateau emission is $10^{43} \mathrm{erg}$ $\mathrm{s}^{-1} \lesssim L_{\text {iso,PL }} \lesssim 10^{47} \mathrm{erg} \mathrm{s}^{-1}$. The duration of the plateau emission is $T_{\mathrm{PL}} \sim 10^{4}-10^{5} \mathrm{~s}$. For comparison, Figures 2 (C) and (D) show only the distributions for redshift-known bursts. There is no clear difference between the distributions for all and redshift-known bursts.

In Figures 2 (E-H), we show the luminosity and duration distributions to compare between bursts with both components (panels E and F) and with single component (panels $\mathrm{G}$ and $\mathrm{H}$ ). For the plateau emission, there seems to be some differences that the luminosity and duration 
Bimodal Long-Lasting Components in Short GRBs
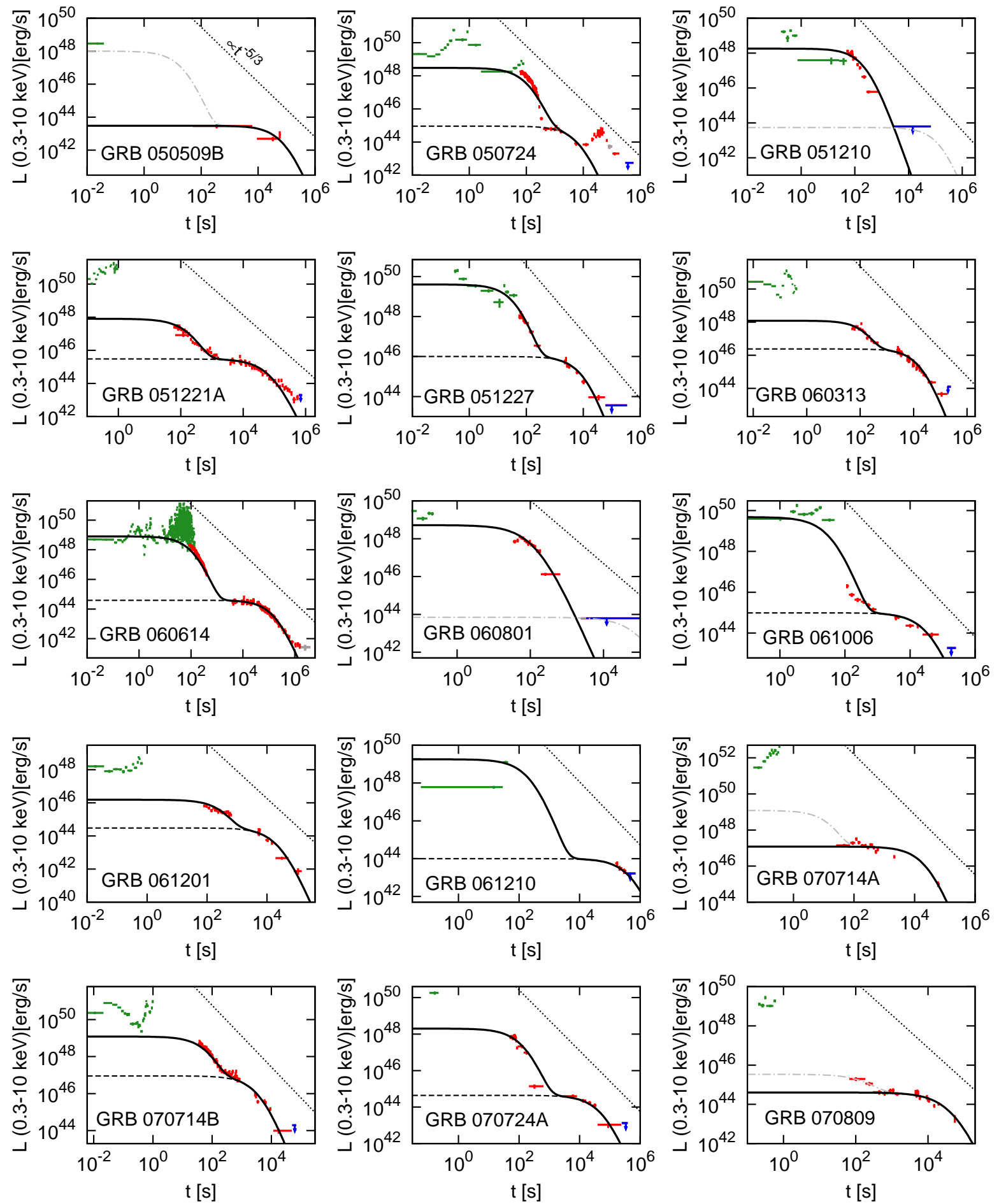

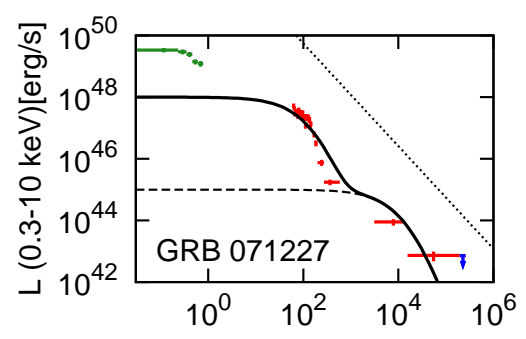

$\mathrm{t}[\mathrm{s}]$

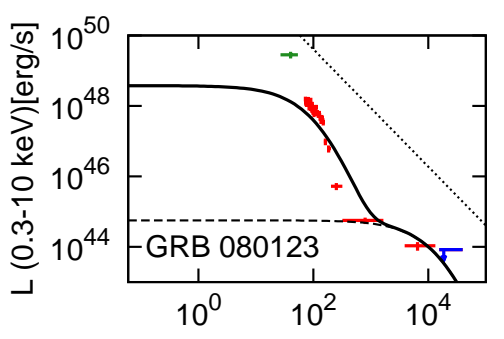

$\mathrm{t}[\mathrm{s}]$

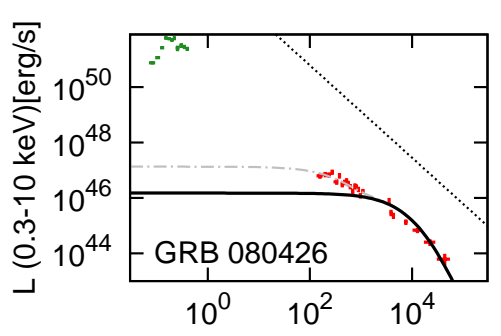

$\mathrm{t}[\mathrm{s}]$

FIG. 1.- Model light curves for the extended and plateau emission of short GRBs as a function of the rest-frame time since the Swift/BAT trigger. Observational data is obtained from UK Swift Science Data Centre. Green and red points are the measured values by Swift/BAT and XRT, respectively. Gray points are the measured values with the exposure time less than 0.1 times the duration error. Blue arrows are the upper limits on the luminosity. We do not fit the data at $t \leq 2 \mathrm{~s}$, which is considered as the prompt emission. Gray dot-dashed curves show the possible components that were missed in the observations. 
Kisaka, Ioka \& Sakamoto

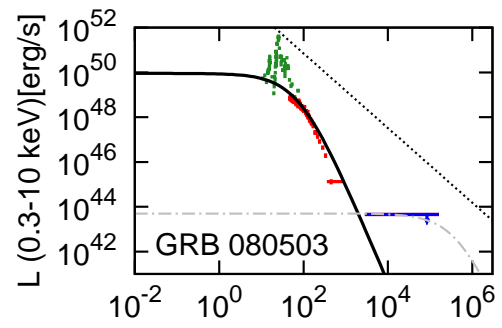

$\mathrm{t}$ [s]

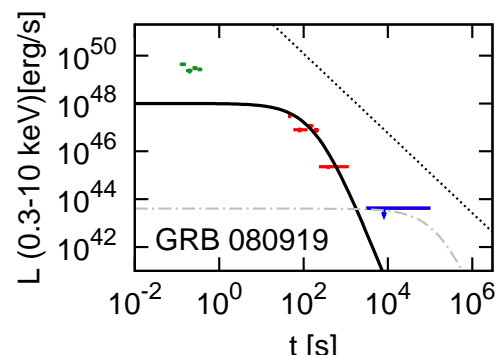

$\mathrm{t}$ [s]

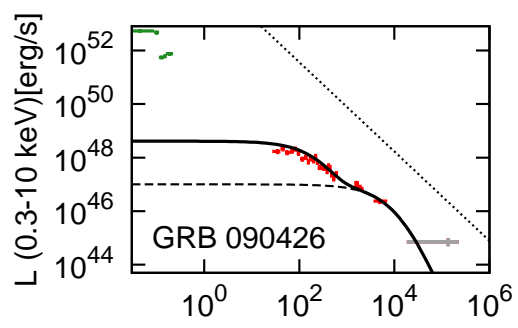

t [s]

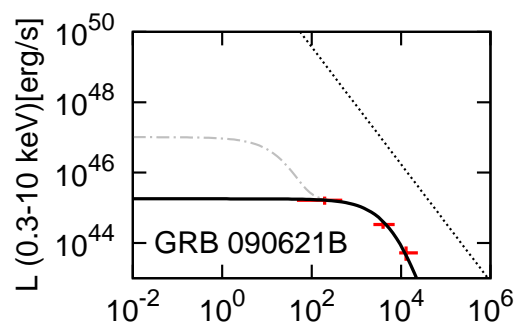

t [s]
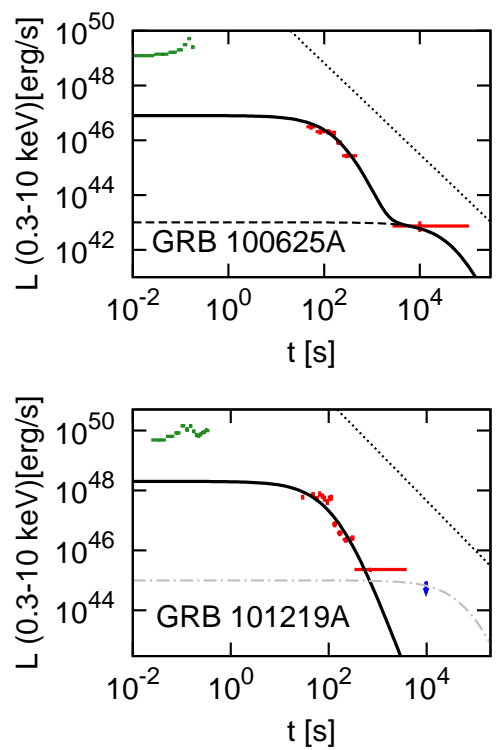

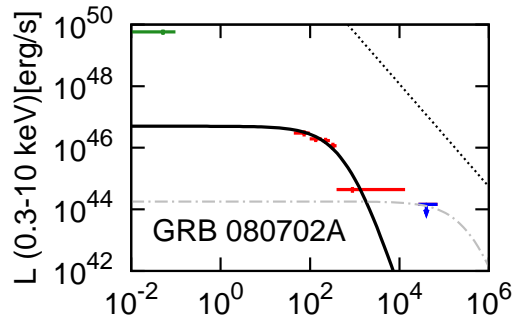

t [s]

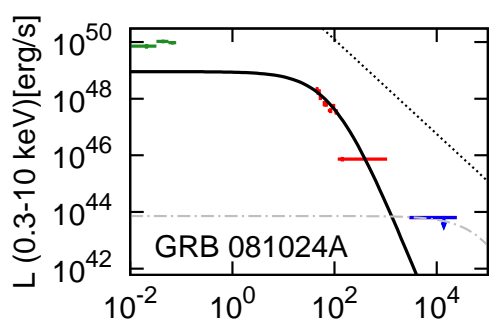

$\mathrm{t}$ [s]

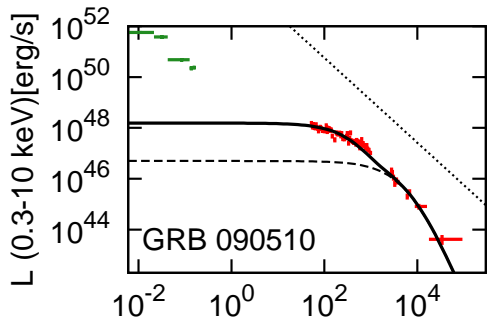

t [s]

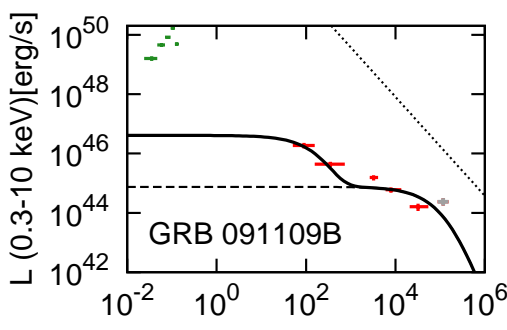

t [s]
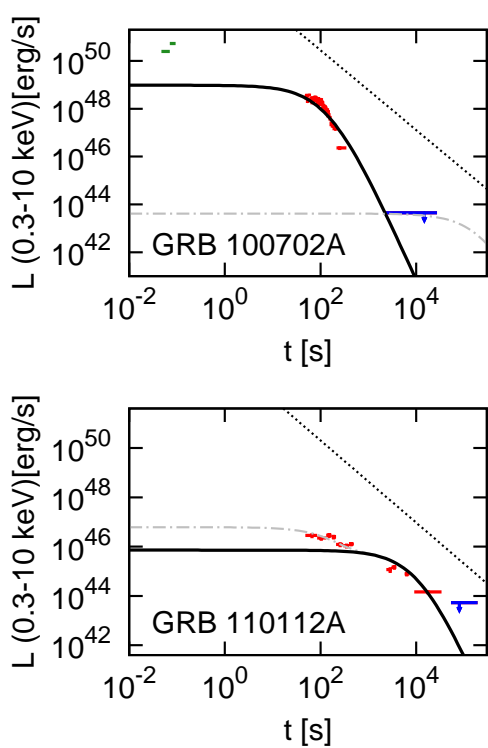

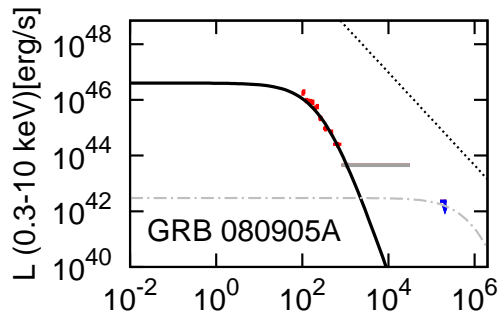

$\mathrm{t}$ [s]
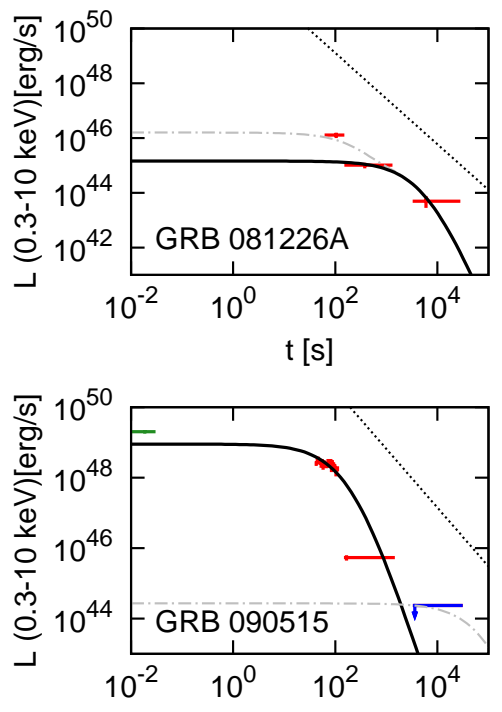

$\mathrm{t}$ [s]

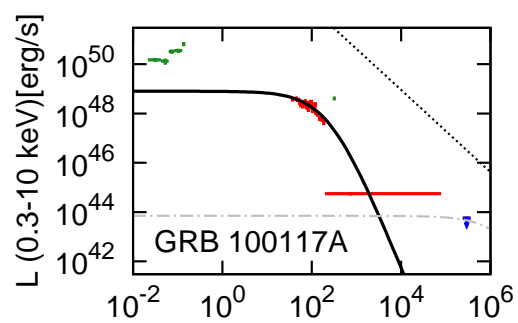

$\mathrm{t}$ [s]
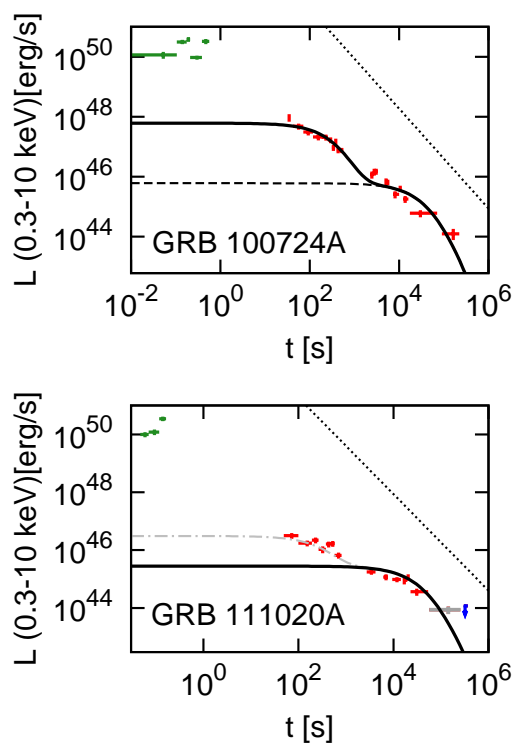

Fig.1- continued. 

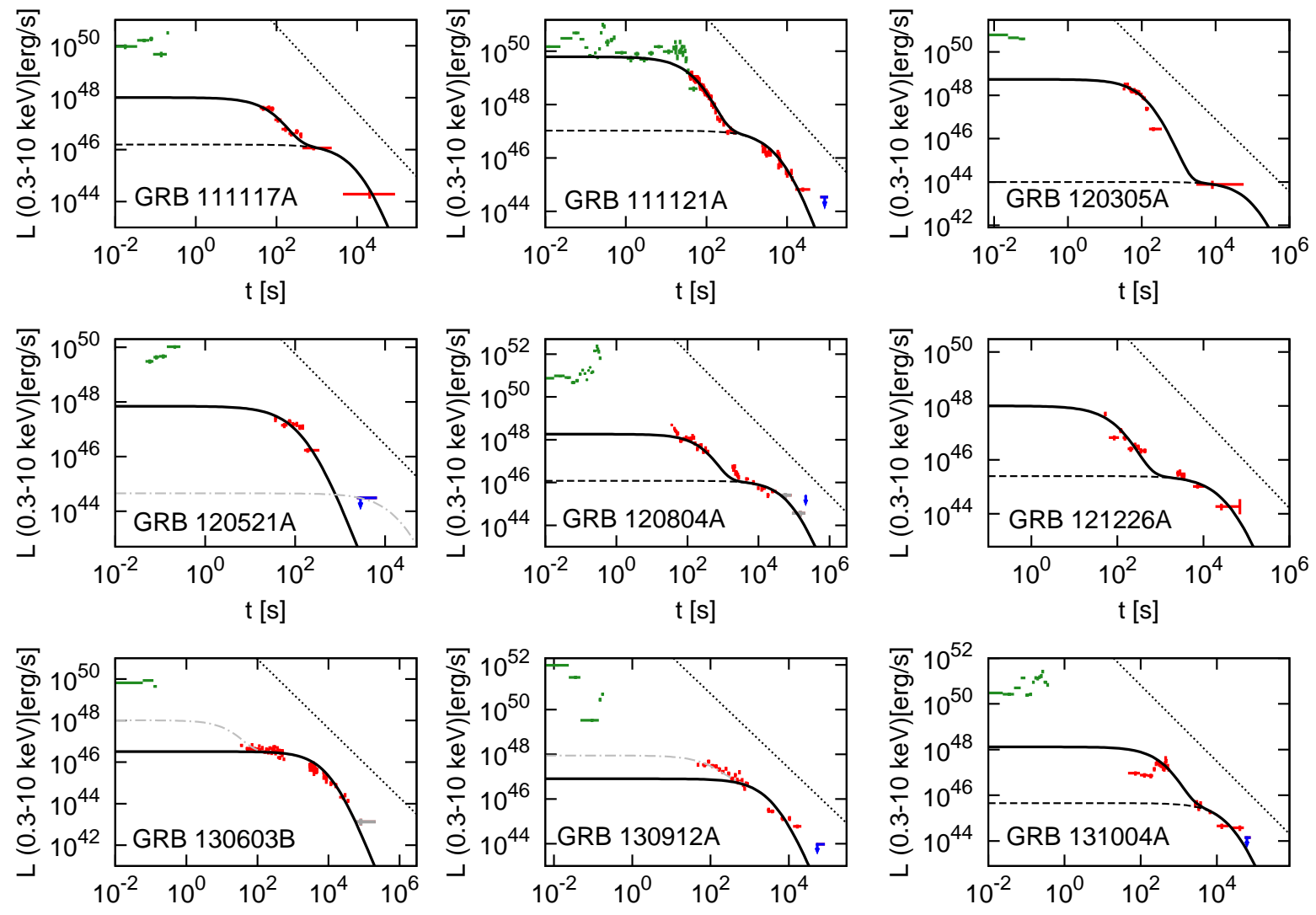

$\mathrm{t}[\mathrm{s}]$

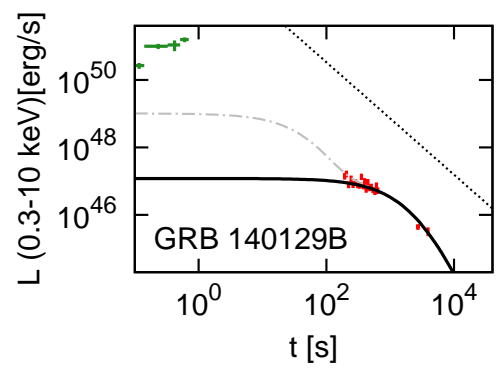

t [s]

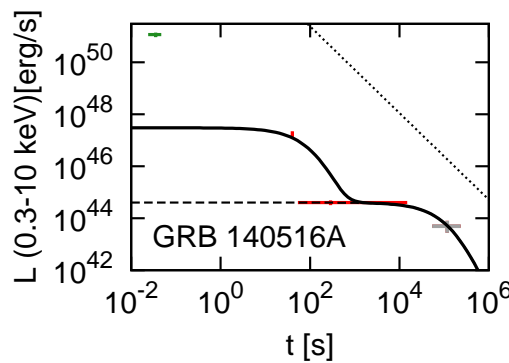

$\mathrm{t}$ [s]
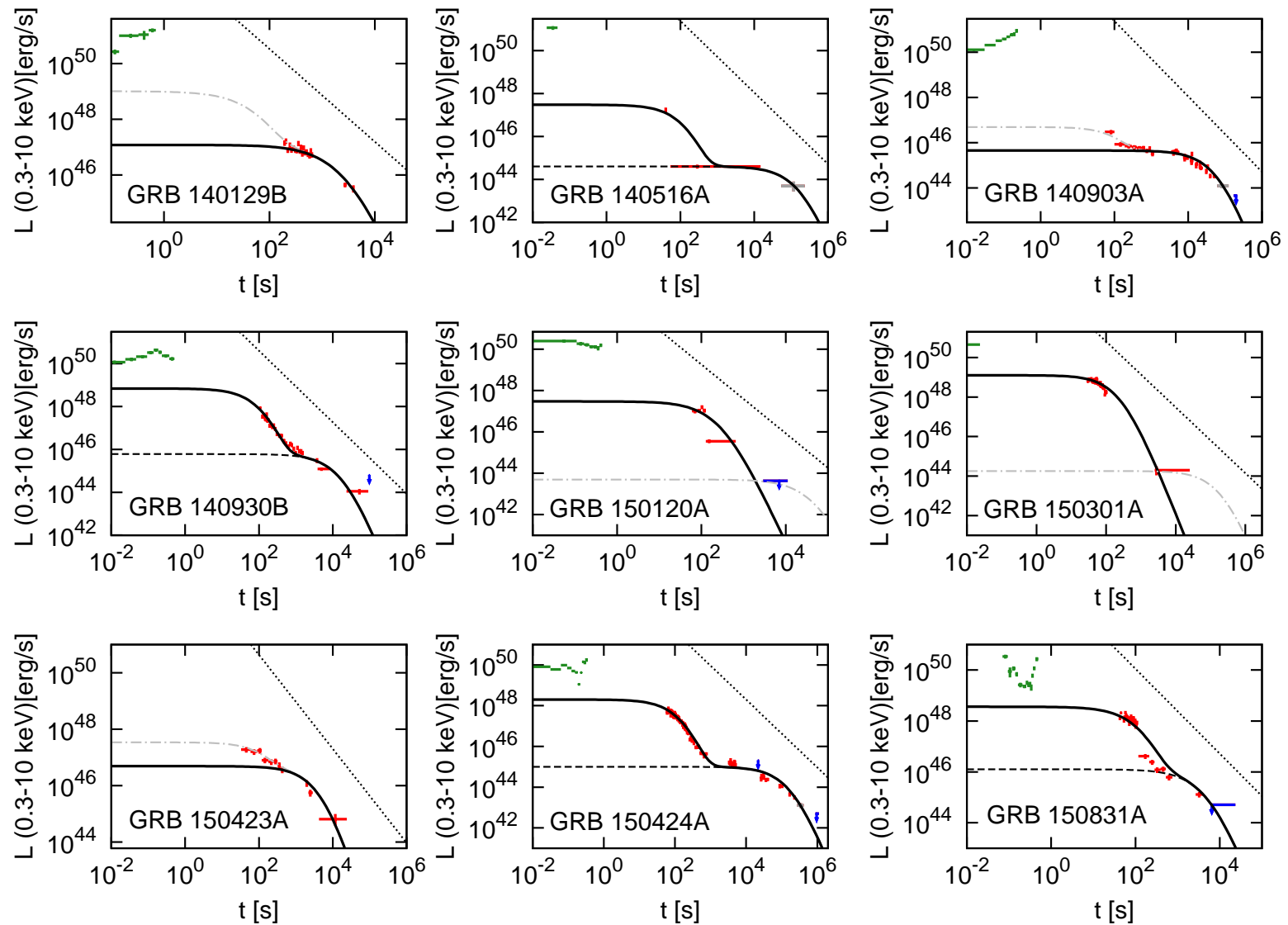

Fig.1- continued. 

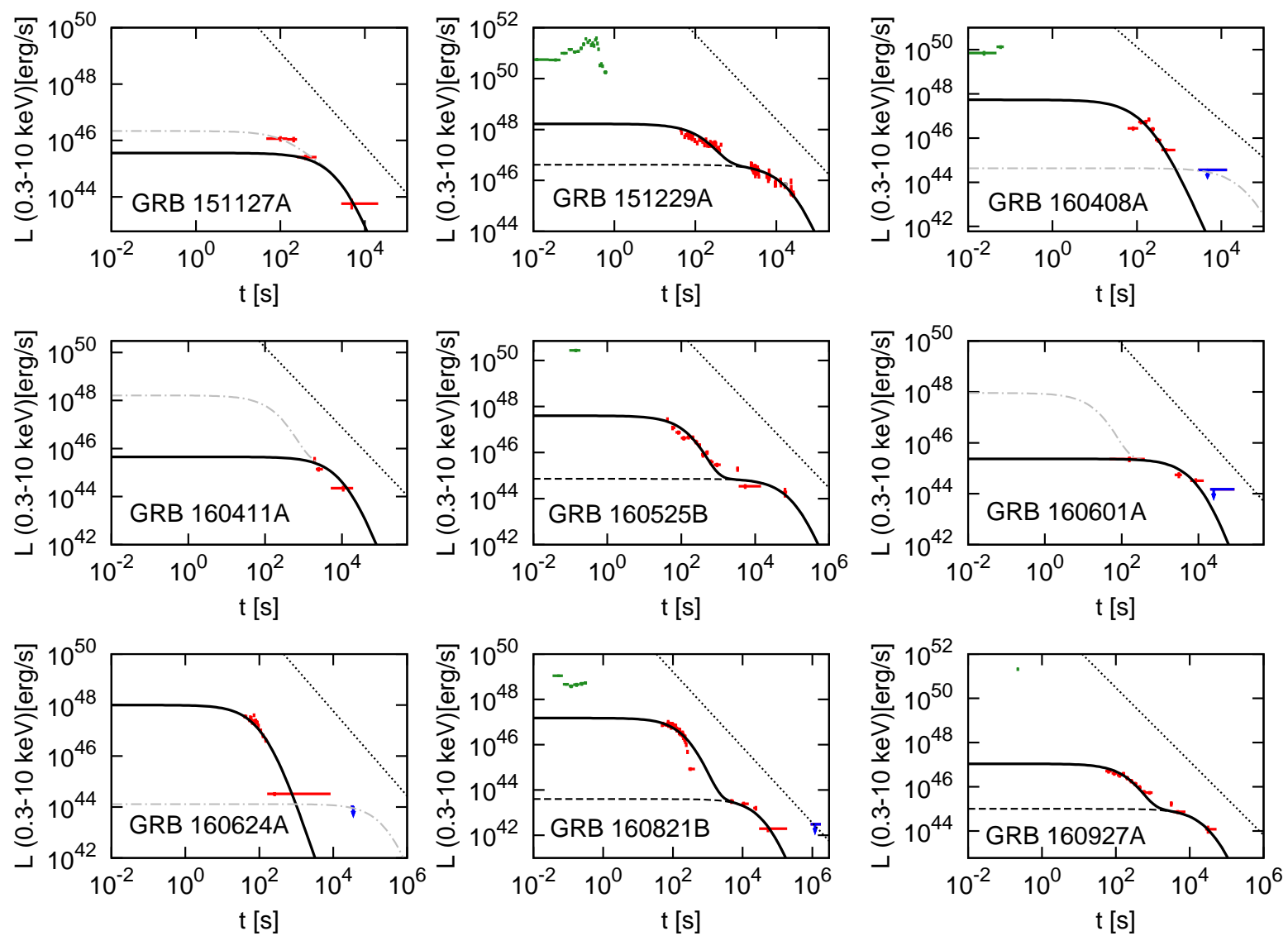

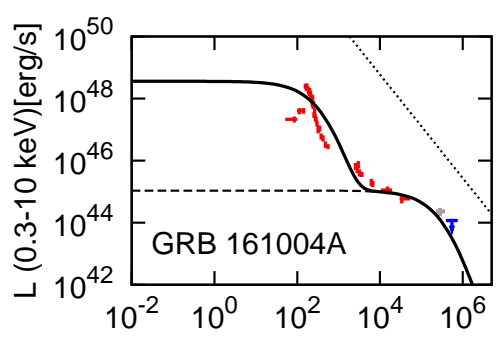

$\mathrm{t}$ [s]

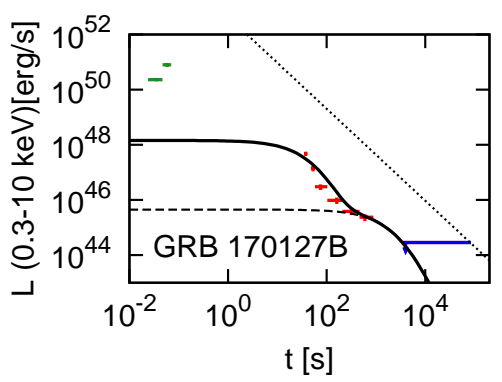

Fig.1- continued. 

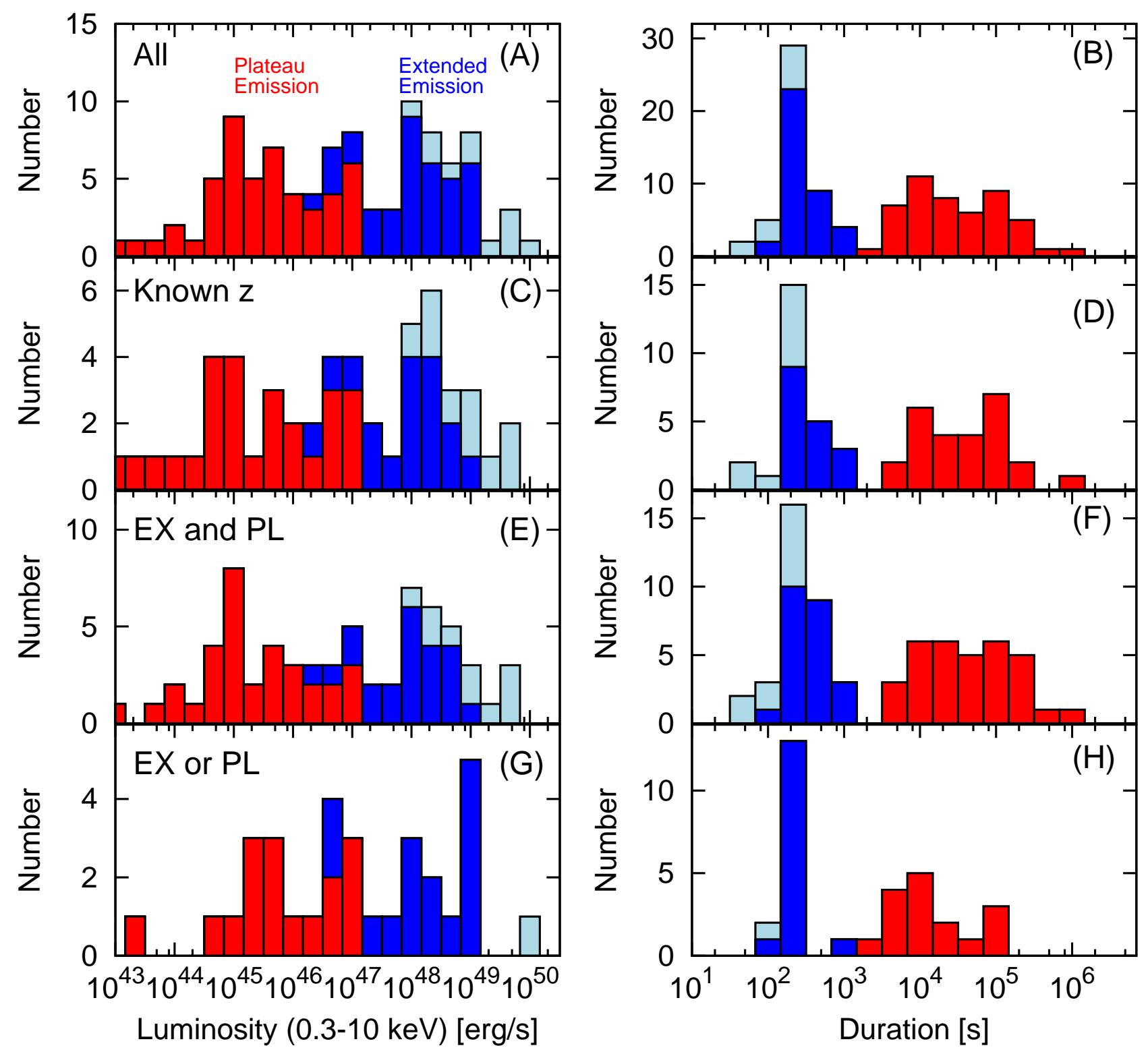

FIG. 2.- Distributions of the luminosity (0.3-10 keV; left) and duration (right) for the Swift/BAT-detected (light-blue) and BAT-nondetected extended emission (blue), and plateau emission (red) in the rest-frame. From top to bottom, panels show the distributions for all bursts $(\mathrm{A}$ and $\mathrm{B})$, bursts with the measured-redshift $(\mathrm{C}$ and $\mathrm{D})$, bursts with both the extended and plateau emission components (E and $\mathrm{F})$, and bursts with single emission component ( $\mathrm{G}$ and $\mathrm{H})$.

of the bursts without the detectable extended emission are respectively high and short on average. A possible bias is that the exposure time of Swift/XRT is set to be too short to detect the low-luminosity and long-duration plateau emission for bursts without detectable extended emission.

Figure 3 shows the luminosity-luminosity (A) and duration-duration plots (B) for the extended and plateau emission obtained from the fitting of the observed light curves. Plotted data are only short GRBs with both the extended and plateau emission. Because of our criterion for the extended emission $\left(L_{\text {iso, } \mathrm{EX}} / L_{\text {iso, PL }}>10\right)$, there is no event at the upper left region from the dashed line, $L_{\text {iso, } \mathrm{PL}}=0.1 L_{\text {iso, EX }}$, in Figure $3(\mathrm{~A})$. Although a weak positive trend may be seen in Figure 3 (A), there is a significant scatter in the distribution. For the durations, no significant correlation is seen in Figure 3 (B). Therefore, it is difficult to predict the luminosity and duration of the plateau emission with an accuracy of a factor of a few from those of the extended emission.

Figure 4 shows the luminosity - duration plot of the extended and plateau emission for all bursts of our sample. A notable feature is that two parameter regions of the extended and plateau emission in Figure 4 are clearly separated. To quantify this finding, we apply model-based cluster analysis to the data using 'mclust' CRAN package (Fraley \& Raftery 2002) of the R software. The luminosity - duration data can best be classified into two groups, which correspond to the clusters of the extended emission (blue of Figure 4) and the plateau 

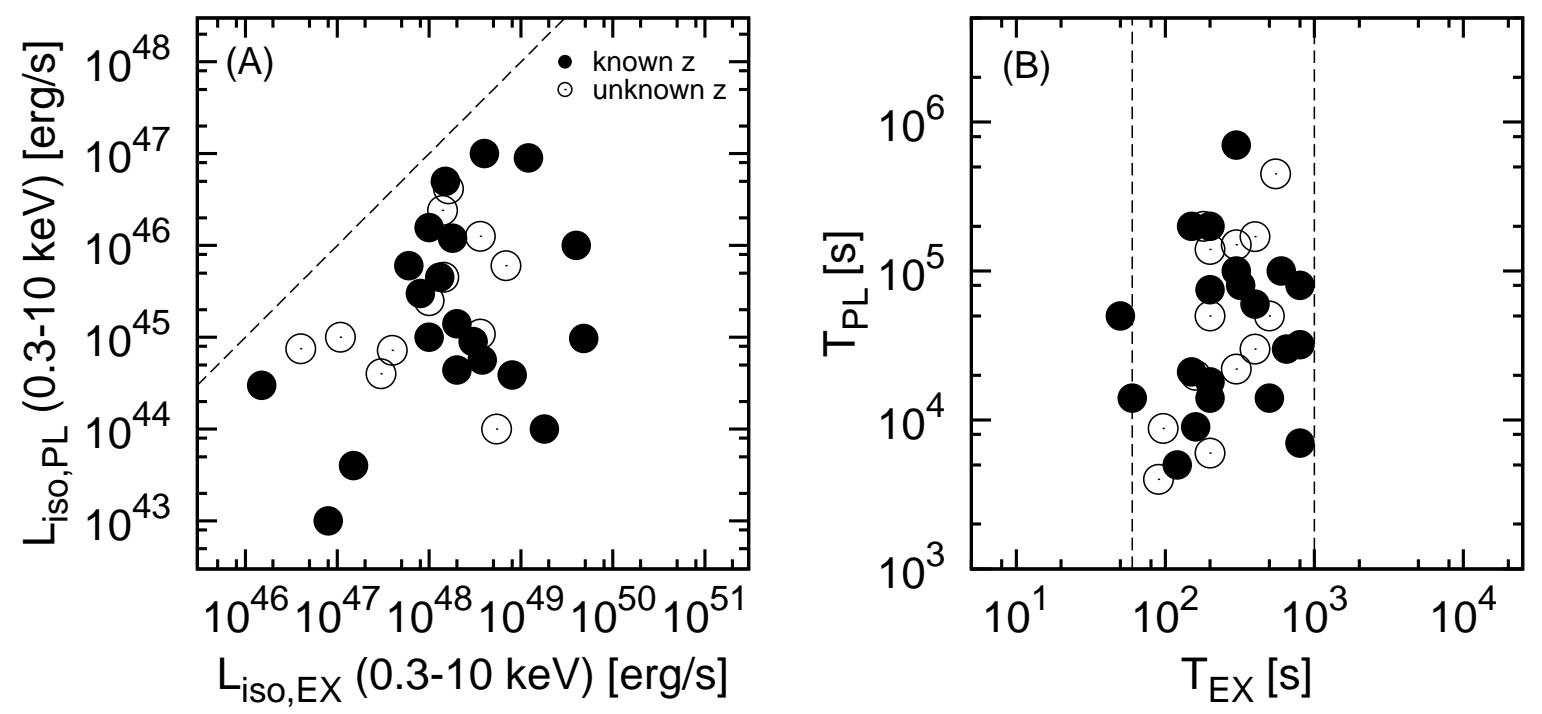

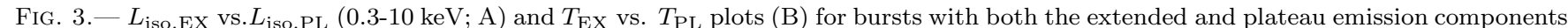
in the rest-frame. Filled symbols are bursts with known redshift, and open symbols are bursts without measured redshift. Dashed line in

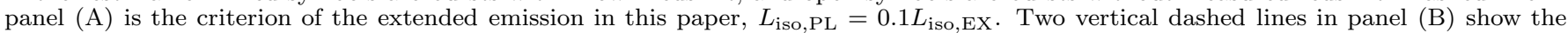
typical starting time of the Swift/XRT observations $(\sim 60 \mathrm{~s})$ and the maximum timescale of the extended emission in our criterion $\left(10^{3} \mathrm{~s}\right)$.

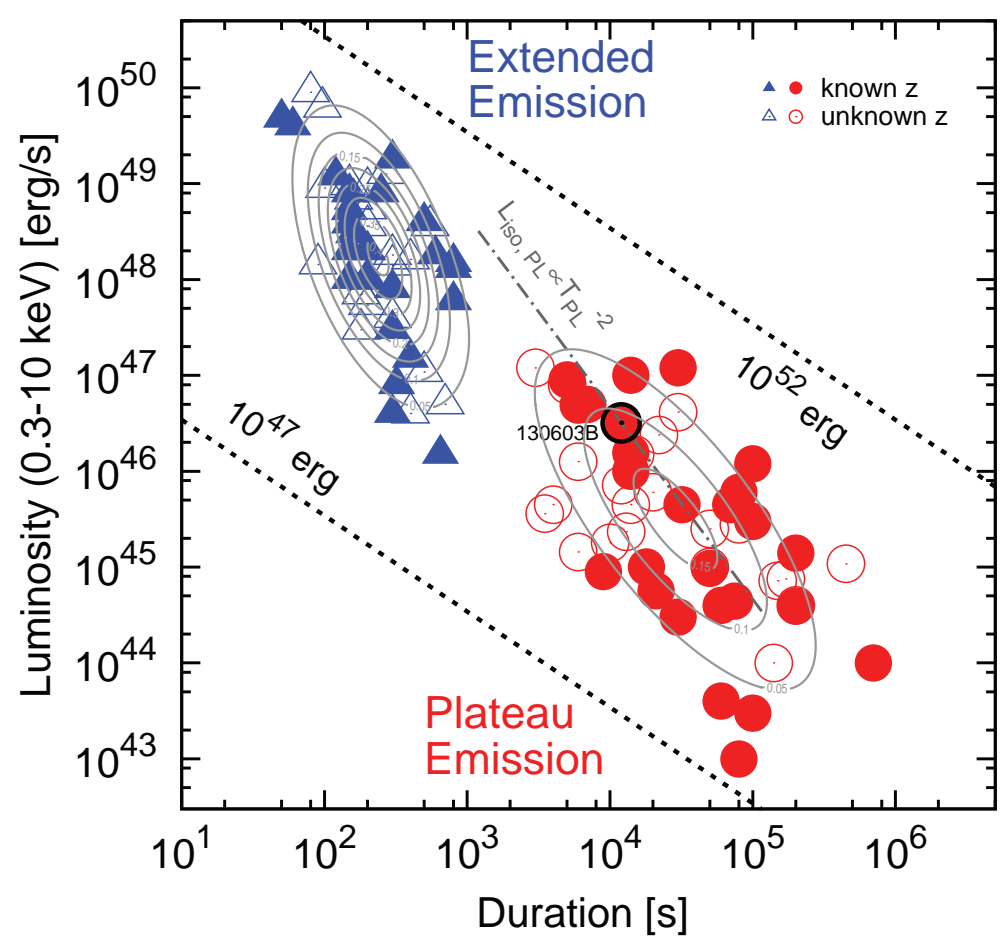

FIG. 4.- Luminosity (0.3-10 keV) vs. duration plot of the extended (blue) and plateau emission (red) in the rest-frame. Filled symbols are bursts with known redshift, and open symbols are bursts without measured redshift. The contours show the density estimate results in the cluster analysis. The null hypothesis that the data are represented by a unimodal distribution is rejected at $>5.2 \sigma$. Gray dot-dashed line shows the relation $L_{\mathrm{iso}, \mathrm{PL}} \propto T_{\mathrm{PL}}^{-2}$ (see Section 4). 
emission (red of Figure 4). The significance of this bimodality is evaluated by the bootstrap approach using 'mclustBootstrapLRT' program which is a part of the 'mclust' package. Based on this analysis, the null hypothesis that the data are represented by a unimodal distribution is rejected at $>5.2 \sigma$. Therefore, our result strongly supports that the extended and plateau emission are distinct components from each other. There is a general trend that the plateau emission with lower luminosity has longer duration (Figure 4), although there is a large scatter. From the light curve formula in Equation (11), the isotropic energy for each component $i(=\mathrm{EX}$ or $\mathrm{PL}$ ) in $0.3-10 \mathrm{keV}$ is

$$
E_{\text {iso }, \mathrm{i}}=\frac{L_{\text {iso }, \mathrm{i}} T_{\mathrm{i}}}{\alpha-1} \quad(\alpha>1) .
$$

Using the value $\alpha=40 / 9$, the isotropic energy is $E_{\text {iso }, \mathrm{i}}=(9 / 31) L_{\mathrm{iso}, \mathrm{i}} T_{\mathrm{i}}$. From Figure 4, the ranges of the isotropic energies of the extended and plateau emission are $10^{48}$ erg $\lesssim E_{\text {iso,EX }} \lesssim 10^{51}$ erg and $10^{47}$ erg $\lesssim$ $E_{\text {iso,PL }} \lesssim 10^{51}$ erg, respectively.

Figure 5(A) plots the energies $E_{\text {iso,EX }}$ and $E_{\text {iso,PL }}$ for short GRBs with both components. The ratio of the energy is $E_{\text {iso,EX }} / E_{\text {iso,PL }} \sim 1-100$. Figure 5 (A) indicates that there is a possible positive trend between two energies $E_{\text {iso,EX }}$ and $E_{\text {iso,PL }}$ for all and redshift-measured bursts (filled circles).

Figures 5 (B) and (C) show the isotropic energy plots of $E_{\text {iso,EX }}$ and $E_{\text {iso,PL }}$ relative to that of the prompt emission, $E_{\mathrm{iso}, \mathrm{PR}}$. We use the $S$ wift/BAT fluence (15$150 \mathrm{keV}$ ) taken from the Swift GRB Table 9 to calculate $E_{\text {iso,PR }}$. For the bursts with the Swift/BATdetected extended emission, we use the fluence of only a short pulse in the BAT energy band for $E_{\text {iso,PR }}$ (Data are provided by A. Lien for GRBs listed in Table 3 of Lien et al. 2016). For GRB 060614, we use the fluence of the short pulse from Gehrels et al. (2006). For GRB 080123, since the extended emission in the Swift/BAT band was weak (Lien et al. 2016), we use the total fluence from the Swift GRB Table as the fluence of the prompt emission. Note that the observed peak energy of the prompt emission of short GRBs $E_{\text {peak }}^{\text {obs }}$ could be higher than $150 \mathrm{keV}$, and the photon index $\alpha_{\mathrm{p}}$ is typically $\sim-1$ (e.g., Lien et al. 2016). In the case of $E_{\text {peak }}^{\text {obs }}>150 \mathrm{keV}$, the bolometric isotropic energy could be $\sim\left(E_{\text {peak }}^{\text {obs }} / 150 \mathrm{keV}\right)^{2+\alpha_{\mathrm{p}}}$ times higher than that in 15 $150 \mathrm{keV}$ band.

Figure 5 (B) shows that the energies of the prompt and extended emission are almost comparable, $E_{\text {iso,PR }} \sim$ $E_{\text {iso,Ex. }}$ The short GRBs with the Swift/BATdetected extended emission have a similar trend (e.g., Perley et al. 2009). This also supports that both the extended emission detected by Swift/BAT and XRT are the same component. For the plateau emission, although the isotropic energy is on average $E_{\text {iso,PL }} \sim 0.1 E_{\text {iso,PR, }}$, a significant fraction of bursts has $E_{\text {iso,PL }}$ comparable to $E_{\text {iso,PR }}$ as shown in Figure 5] (C). This would suggest that the plateau emission is also produced by the central engine activities. The energy $E_{\text {iso,PL }}$ also seems to have a positive trend with $E_{\text {iso,PR }}$.

\footnotetext{
${ }^{9}$ https://swift.gsfc.nasa.gov/archive/grb_table/
}

\section{DETECTABILITY AS ELECTROMAGNETIC COUNTERPARTS TO GRAVITATIONAL WAVE SOURCES}

The leading model of short GRBs is an NS binary merger (e.g., Narayan et al. 1992). The NS binary merger is accompanied with strong GW emission, which can be detected by current GW detectors. A simultaneous detection of GW and EM emission would maximize the available information from this spectacular event (e.g., Metzger \& Berger 2012).

Current $\gamma$-ray detectors such as Swift/BAT can detect the prompt emission if the short GRB occurs within the GW detection horizon ( $\sim 100 \mathrm{Mpc})$ and the GRB jet points to us. Since the duration of the prompt emission is $\lesssim 1 \mathrm{~s}$, the detection probability is mainly determined by the field-of-view of the detectors.

The long-lasting components, the extended and plateau emission, are also bright enough for detections if a short GRB occurs within the GW detection horizon. By virtue of the long duration, the follow-up observations are possible after receiving the GW detection alert (within $\lesssim 10^{2}$ s; Singer et al. 2014; Chen \& Holz 2015; Singer \& Price 2016). Especially, since the duration of the plateau emission is longer than the orbital period of all-sky survey detectors, the detection probability is much higher than that determined by the ratio of the field-of-view to the all-sky.

Nearly isotropic emission is also expected. A significant fraction of the plateau emission could be scattered to a wide solid angle by the merger ejecta (Kisaka et al. $2015 \mathrm{~b})$. During the plateau activity timescale $\left(\sim 10^{4} \mathrm{~s}\right)$, the radius of the plateau emission region is smaller than that of the expanding merger ejecta. The optical depth for the Thomson cross section is typically larger than unity during the plateau phase. Since the Lorentz factor is low $(\Gamma \sim 10)$ inside the jet due to the cocoon confinement (Bromberg et al. 2011; Nagakura et al. 2014), the relativistic beaming angle is larger than the jet opening angle $\theta_{\mathrm{j}}$. Then, the emitted photons with angle $\gtrsim \theta_{\mathrm{j}}$ relative to the jet axis are scattered to a large angle by the surrounding non-relativistic ejecta (Kisaka et al. $2015 \mathrm{~b})$. Since the collimated emission is scattered to an isotropic distribution, the luminosity of the scattered component is

$$
L_{\text {scatter }} \sim 10^{-3}\left(\theta_{\mathrm{j}}^{2} / 10^{-3}\right) L_{\mathrm{iso}, \mathrm{PL}}
$$

Here, we investigate the detectability of on-axis extended and plateau emission taking into account their luminosity and duration distributions. We also consider the detectability of the scattered plateau emission. Figure 6] shows the energy flux and duration of the extended and plateau emission if the short GRBs in our sample occur at $100 \mathrm{Mpc}$, which is approximately the detection horizon of a binary NS merger for the current GW detectors (Abbott et al. 2016d). We use Equation (3) to estimate the flux of the scattered plateau emission (Kisaka et al. 2015b). In Figure 6, we also plot the flux sensitivities of the current and planned X-ray detectors. Since the typical photon index of the extended emission is $\sim-2$ (Lien et al. 2016) and a similar value within the uncertainty for the plateau emission (according to 

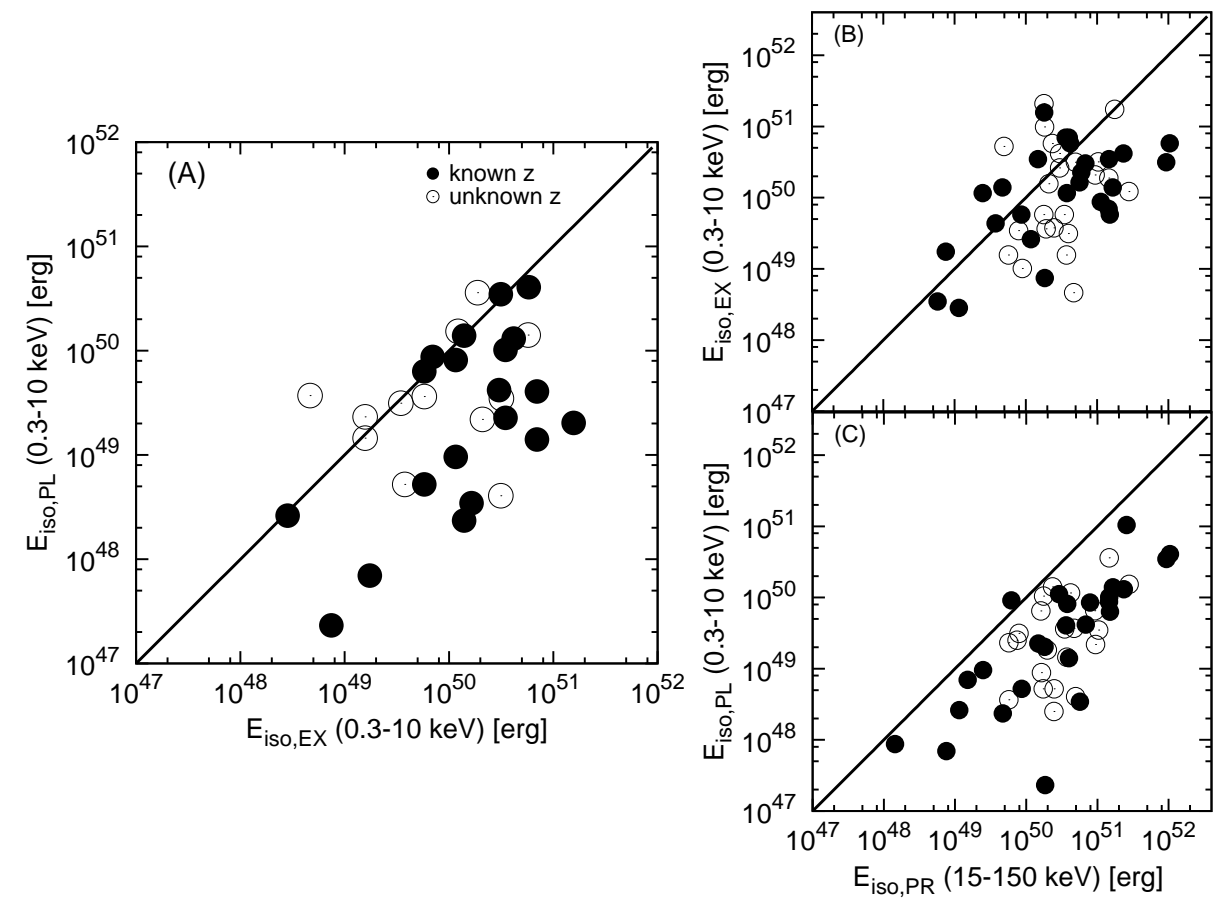

FIG. 5.- Plots of the isotropic energies $E_{\text {iso,PL }}(0.3-10 \mathrm{keV}), E_{\text {iso,Ex }}(0.3-10 \mathrm{keV})$ and $E_{\text {iso,PR }}(15-150 \mathrm{keV})$. Filled circles are bursts with known redshift, and open circles are bursts without measured-redshift.

Swift/XRT GRB light curve repository [10), we neglect the difference of the energy bands for each detector to consider the energy flux.

From Figure 6, the energy flux distributions of the extended and plateau emission are $\sim 10^{-7}-10^{-4} \mathrm{erg}$ $\mathrm{cm}^{-2} \mathrm{~s}^{-1}$ and $\sim 10^{-11}-10^{-7} \mathrm{erg} \mathrm{cm}^{-2} \mathrm{~s}^{-1}$, respectively. The monitoring observations by CALET/HXM, Fermi/GBM, INTEGRAL/SPI-ACS, and Swift/BAT can detect most of the extended emission population if the line of sight is within the jet opening angle. Swift/BAT and MAXI/GSC can detect some bright plateau emission. Note that MAXI scans the entire sky every 92-minute orbital period, which is comparable to or shorter than the duration of the plateau emission. Although eROSITA can detect most of the scattered plateau emission, its small field-of-view (0.833 $\mathrm{deg}^{2}$ ) makes it difficult to detect the X-ray signals with GW simultaneously.

In the follow-up observations of the GW sources, Swift/XRT with $100 \mathrm{~s}$ integration time Kanner et al. 2012), future ISS-Lobster/WFI with $450 \mathrm{~s}$ integration time (Camp et al. 2013), and future Einstein Probe/WXT with $1000 \mathrm{~s}$ integration time (Yuan et al. 2015) can detect the plateau emission. These detectors can also detect the scattered plateau emission in the bright population including GRB 130603B (Figure 6).

The long-lasting activities of the central engine could significantly contribute to the heating of the merger ejecta (engine-powered macronova; Kulkarni 2005; Yu. Zhang \& Gao 2013; Metzger \& Piro 2014; Kisaka et al. 2015a,b). The emission from the heated

\footnotetext{
10 http://www.swift.ac.uk/xrt_curves/
}

ejecta is observed as a macronova and has been discussed as a promising EM counterpart to the NS binary merger (e.g., Li \& Paczvńsky 1998; Kulkarni 2005; Metzger et al. 2010b; Kisaka et al. 2015a,b, 2016). The observed peak luminosity depends on the internal energy in the ejecta at the time when the diffusion timescale of photons in the ejecta becomes comparable to the dynamical timescale. If the energy injection timescale is earlier than the peak phase of the macronova, the internal energy in the ejecta decreases due to the adiabatic cooling. Then, the plateau emission is more effective for heating than the extended emission (Kisaka et al. 2015b). The internal energy in the heated ejecta after the energy injection $\left(t>T_{\mathrm{PL}}\right)$ is $\sim E_{\mathrm{int}}\left(t / T_{\mathrm{PL}}\right)^{-1} \propto L_{\mathrm{iso}, \mathrm{PL}} T_{\mathrm{PL}}^{2}$, where the total injected energy is determined by the radiative efficiency $\eta$, the jet opening angle $\theta_{\mathrm{j}}$, the observed isotropic luminosity $L_{\text {iso, } \mathrm{PL}}$, and the duration $T_{\mathrm{PL}}$, as $E_{\text {int }}=$ $\left[\left(\theta_{\mathrm{j}}^{2} / 2\right) / \eta\right] L_{\mathrm{iso}, \mathrm{PL}} T_{\mathrm{PL}}$. Note that the value of $L_{\mathrm{iso}, \mathrm{PL}} T_{\mathrm{PL}}^{2}$ for GRB 130603B $\left(L_{\text {iso, PL }} \sim 4 \times 10^{46} \mathrm{erg} \mathrm{s}^{-1}, T_{\mathrm{PL}} \sim\right.$ $10^{4} \mathrm{~s}$ ), which was first reported as a macronova event (Tanvir et al. 2013; Berger et al. 2013a), lies in the median of the distribution of $L_{\mathrm{iso}, \mathrm{PL}} T_{\mathrm{PL}}^{2}$ (gray dot-dashed line in Figure (4). Thus the peak luminosity of the macronova associated with GRB 130603B is a typical value of the engine-powered macronova, implying that the dominant energy source could be the central engine not the radioactivity of $r$-process elements.

\section{IMPLICATIONS FOR BH ENGINE MODELS}

In Section 3, we show that the light curve of most short GRBs consists of bimodal long-lasting components following the prompt emission. The plateau-like evolution 


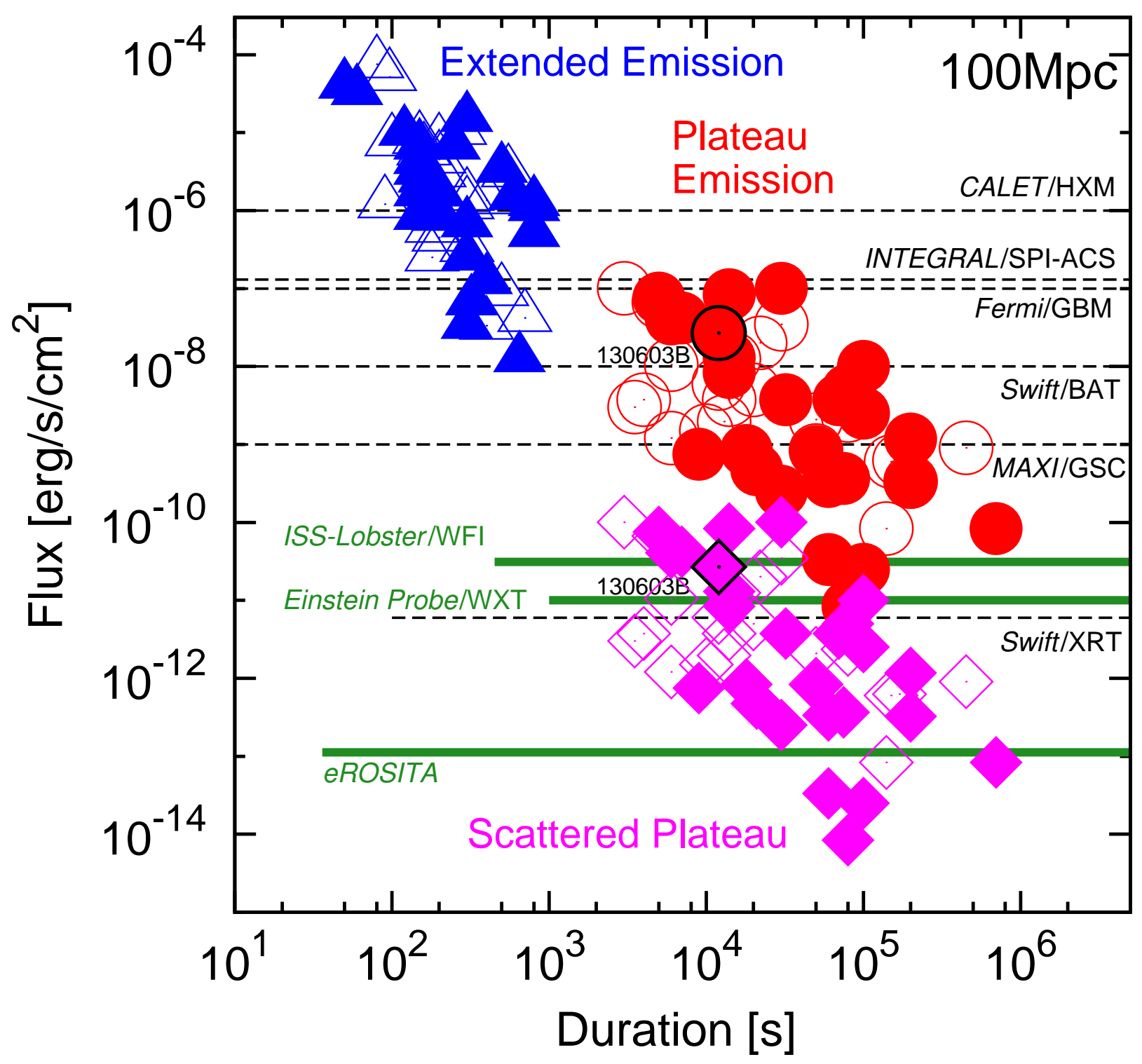

FIG. 6.- Flux-duration plot of extended, plateau and scattered emission components for all short GRBs with distance 100 Mpc. Black dashed lines show the sensitivity limits of $C A L E T / \mathrm{HXM}$ (integration time $1 \mathrm{~s}$; Adriani et al. 2016), INTEGRAL/SPI-ACS (integration time $1 \mathrm{~s}$; Savchenko et al. 2016), Fermi/GBM (integration time $1 \mathrm{~s}$; Naravana Bhat et al. 2016), Swift/BAT (integration time 1 s; Krimm et al. 2013), Swift/XRT (integration time $100 \mathrm{~s}$; Kanner et al. 2012), and MAXI/GSC (integration time corresponding to a single pass; Sugizaki et al. 2011). Green solid lines show the sensitivity limits of planned detectors, ISS-Lobster/WFI (integration time $450 \mathrm{~s}$; Camp et al. 2013), Einstein Probe/WXT (integration time $1000 \mathrm{~s}$; Yuan et al. 2015), and eROSITA (integration time corresponding to a single survey pass; Merloni et al. 2012).

$\left(L \propto t^{0}\right)$ is difficult to explain by the normal afterglow model (e.g., Sari et al. 1998). For a single plateau model such as the spin-down of highly magnetized NSs (e.g., Usov 1992; Zhang \& Mészáros 2001; Gao \& Fan 2006; Metzger et al. 2008b; Bucciantini et al. 2012; Lyutikov 2013) and BHs (e.g., Barkov \& Pozanenko 2011; Nakamura et al. 2014), additional mechanisms to produce another component are required (Gompertz et al. 2014; Gibson et al. 2017).

The phenomenological light curve in Equation (1) is motivated by the $\mathrm{BH}$ engine model Kisaka \& Ioka 2015). An NS-NS or BH-NS merger leaves a BH with a surrounding disk and merger ejecta (e.g.,
Hotokezaka et al. 2013; Kvutoku et al. 2015). In this model, a relativistic jet is launched via BlandfordZnajek (BZ) process (Blandford \& Znajek 1977) from the $\mathrm{BH}$. For the $\mathrm{BH}$ with a mass $M_{\mathrm{BH}}$, a spin parameter $a=J c / G M_{\mathrm{BH}}$, an angular frequency $\Omega_{\mathrm{H}}=$ $a c /\left(2 M_{\mathrm{BH}} r_{\mathrm{H}}\right)$, and a magnetic flux $\Psi_{\mathrm{BH}} \sim \pi r_{\mathrm{H}}^{2} B_{\mathrm{H}}$, the total power of the BZ jet is (e.g., Blandford \& Znajek 1977; Tchekhovskoy et al. 2011)

$$
L_{\mathrm{BZ}} \sim \frac{\kappa}{4 \pi c} \Omega_{\mathrm{H}}^{2} \Psi_{\mathrm{BH}}^{2},
$$

where $\kappa \approx 0.05, J$ is the angular momentum of the $\mathrm{BH}$, $B_{\mathrm{H}}$ is the strength of the magnetic field at the $\mathrm{BH}, r_{\mathrm{H}}$ 


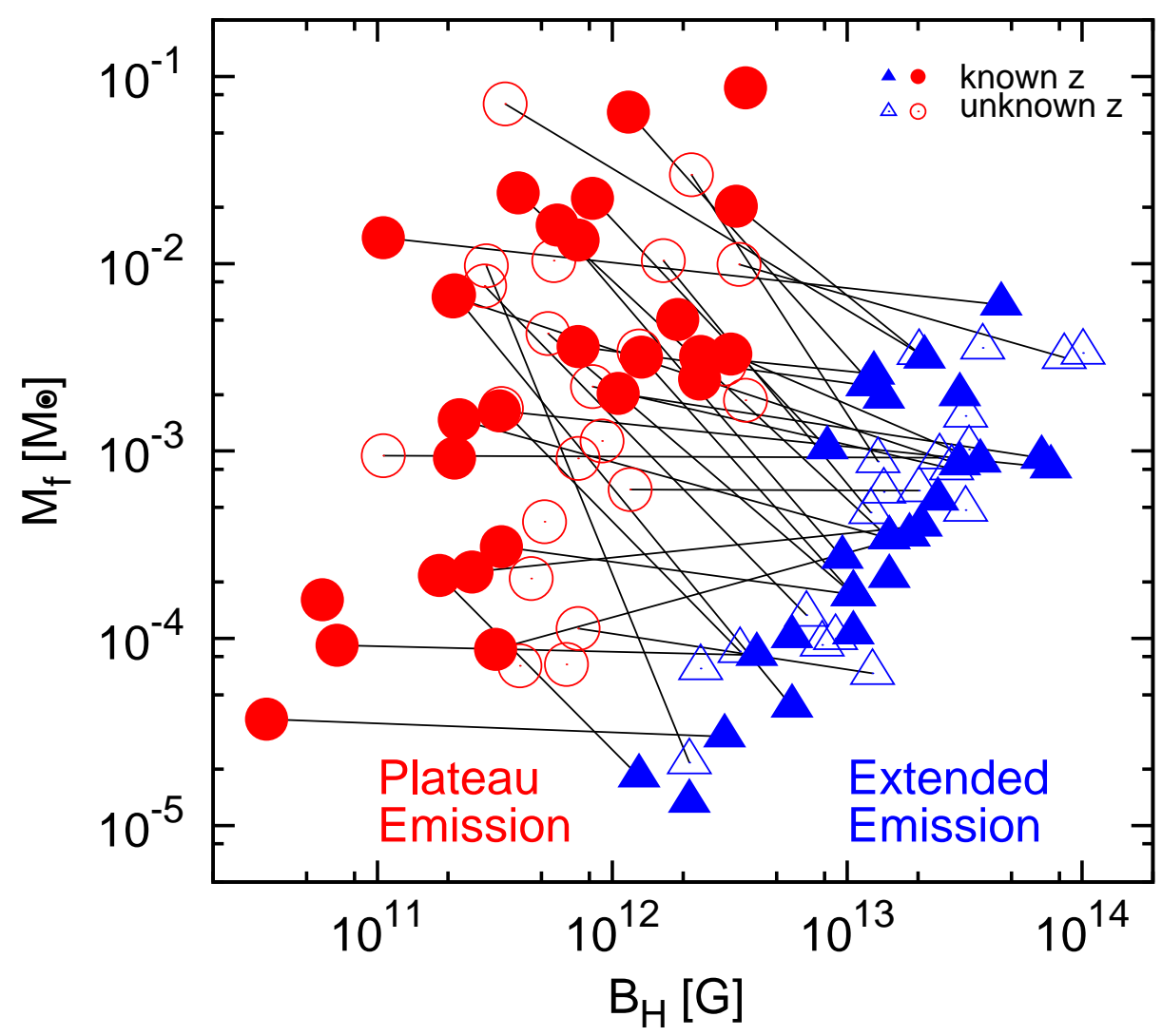

FIG. 7.- Estimated total mass of the fallback matter $M_{\mathrm{f}}$ and magnetic field $B_{\mathrm{H}}$ for extended (blue triangles) and plateau emission (red circles). Filled symbols are events with known redshift, and open symbols are events without measured redshift. Solid lines connect each event of short GRBs.

is the radius of the $\mathrm{BH}$ horizon, $c$ is the light speed, and $G$ is the gravitational constant, We use $a / M_{\mathrm{BH}} \sim 0.7$ as a fiducial value (Shibata \& Taniguchi 2006). Taking into account the beaming correction $\left(\theta_{\mathrm{j}}^{2} \sim 10^{-3}\right.$; Fong et al. 2014, 2015) and the radiative efficiency $(\eta \sim$ 0.1; Zhang et al. 2007), the observed isotropic luminosity is

$$
L_{\text {iso }} \sim \eta\left(2 / \theta_{\mathrm{j}}^{2}\right) L_{\mathrm{BZ}} \sim 10^{2} L_{\mathrm{BZ}} .
$$

As long as the pressure of fallback matter supports the magnetic flux on the $\mathrm{BH}$, the $\mathrm{BZ}$ power remains flat, $L_{\text {iso }} \propto t^{0}$ (see also Tchekhovskoy \& Giannios 2015).

The timescale of each component $T_{\mathrm{i}}$ is determined by the pressure balance between the fallback matter and magnetic field. Such a disk state is the so-called magnetically arrested disk (e.g., Narayan et al. 2003). If the matter pressure cannot support the magnetic flux on $\mathrm{BH}$, the BZ power reduces. The temporal evolution of the mass accretion rate is (e.g., Rosswog 2007; Kyutoku et al. 2015),

$$
\dot{M}=\frac{2}{3} \frac{M_{\mathrm{f}}}{0.1 \mathrm{~s}}\left(\frac{t}{0.1 \mathrm{~s}}\right)^{-5 / 3}, \quad(t>0.1 \mathrm{~s}),
$$

where $M_{\mathrm{f}} \equiv \int_{0.1 \mathrm{~s}}^{\infty} \dot{M} d t$ is the total fallback mass after the reference time $t>0.1 \mathrm{~s}$. From the force balance between the matter and magnetic field pressures, the characteris- tic timescale of the BZ jet is given by

$$
T \sim 1 \times 10^{4}\left(\frac{M_{\mathrm{f}}}{10^{-3} M_{\odot}}\right)^{3 / 5}\left(\frac{B_{\mathrm{H}}}{10^{12} \mathrm{G}}\right)^{-6 / 5} \mathrm{~s},
$$

where we use the $\mathrm{BH}$ mass $M_{\mathrm{BH}}=3 M_{\odot}$, and the radial velocity $v_{\mathrm{R}} \sim 10^{-2} \sqrt{G M_{\mathrm{BH}} / R_{\mathrm{H}}}$ (e.g., Tchekhovskoy et al. 2011; Zamaninasab et al. 2014).

We discuss the implications for the $\mathrm{BH}$ engine model (Kisaka \& Ioka 2015) from the results in Section 3. Since the light curve implied by the $\mathrm{BH}$ engine model is consistent with the observations, the results in Kisaka \& Ioka (2015) are also applicable to our sample. Using the obtained parameters $L_{\text {iso,EX }}, L_{\text {iso,PL }}, T_{\mathrm{EX}}$, and $T_{\mathrm{PL}}$, we can estimate the magnetic field $B_{\mathrm{H}}$ and the total fallback mass $M_{\mathrm{f}}$ as follows. The strength of the magnetic field $B_{\mathrm{H}}$ is determined by the observed luminosity $L_{\text {iso }}$ from Equation (15), as

$$
B_{\mathrm{H}} \sim 3 \times 10^{12}\left(\frac{\eta / \theta_{\mathrm{j}}^{2}}{10^{2}}\right)^{-1 / 2}\left(\frac{L_{\text {iso }}}{10^{47} \mathrm{erg} \mathrm{s}^{-1}}\right)^{1 / 2} \mathrm{G}
$$

On the other hand, the total fallback mass $M_{\mathrm{f}}$ is derived from Equations (7) and (8), as

$$
M_{\mathrm{f}} \sim 1 \times 10^{-2}\left(\frac{\eta / \theta_{\mathrm{j}}^{2}}{10^{2}}\right)^{-1}
$$




$$
\times\left(\frac{L_{\text {iso }}}{10^{47} \operatorname{erg~s}^{-1}}\right)\left(\frac{T}{10^{4} \mathrm{~S}}\right)^{5 / 3} M_{\odot} .
$$

Figure 7 shows the distributions of $B_{\mathrm{H}}$ and $M_{\mathrm{f}}$ from the observations. The ranges of the magnetic field strength are $B_{\mathrm{H}} \sim 10^{12}-10^{14} \mathrm{G}$ for the extended emission, and $B_{\mathrm{H}} \sim 10^{11}-10^{13} \mathrm{G}$ for the plateau emission. The range of the magnetic field for the plateau emission is consistent with that of typical NSs and PSR J07373039B in the double pulsar system (Lvne et al. 2004). The range of the fallback mass $M_{\mathrm{f}} \sim 10^{-5}-10^{-1} M_{\odot}$ is consistent with the numerical relativity simulations (e.g., Hotokezaka et al. 2013; Kvutoku et al. 2015; Kawaguchi et al. 2015; Foucart et al. 2015, 2017). There is significant dispersion in the distribution of the derived fallback mass. For BH-NS mergers, the total mass and mass ratio of the binaries before the merger should have some dispersions, which may explain the dispersion of the fallback mass $M_{\mathrm{f}}$. On the other hand, for the NS-NS mergers, the dispersions of the total mass and the mass ratio are relatively narrow. Then, the large dispersion could stem from the varieties of the radiation efficiency $\eta$ and the jet opening angle $\theta_{\mathrm{j}}$.

Note that the populations of the extended and plateau emission do not overlap in $M_{\mathrm{f}}-B_{\mathrm{H}}$ plot (Figure 7). This is because the distributions of two durations $T_{\mathrm{EX}}$ and $T_{\mathrm{PL}}$ are completely separated (Figure 4). In fact, two populations are split by the line $M_{\mathrm{f}} \propto B_{\mathrm{H}}^{2}$ derived from Equation (7) with $T=$ const. From Equation (7), the timescale does not depend on the radiation efficiency $\eta$ and the jet opening angle $\theta_{\mathrm{j}}$. Then, the populations of the extended and plateau emission do not overlap in Figure 7 irrespective of $\eta$ and $\theta_{\mathrm{j}}$.

In some bursts, the fallback mass calculated from the extended emission is rather different from that from the plateau emission (Figure 7). The duration of extended emission $T_{\mathrm{EX}}$ could become short because of the decrease of the magnetic flux $\Psi_{\mathrm{BH}}$ via the magnetic reconnection (phase VI in Figure 2 of Kisaka \& Ioka 2015). Then, the fallback mass $M_{\mathrm{f}}$ from Equation (9) is underestimated. On the other hand, at the plateau emission phase, the initial magnetic flux of an NS before the merger would give the lower bound on $\Psi_{\mathrm{BH}}$ (phase VII in Figure 2 of Kisaka \& Ioka 2015). Then, the fallback mass estimated from Equation (9) tends to be larger than that for the extended emission. Therefore, it is reasonable that the fallback mass derived from the extended emission tends to be smaller than that from the plateau emission.

In a few bursts, the temporal flux decay at the end of the extended emission seems much steeper than the BH engine model (e.g., Barthelmy et al. 2005). Such steep decays are produced only by the activity of the central engine (Ioka et al. 2005). The light curve model described in Equation (11) and shown in Figure 1 is a simple toy model. Detailed processes such as the magnetic flux decay due to the magnetic reconnection near the $\mathrm{BH}$ horizon are not included in our model, and can produce the short-timescale seen at the end of the extended emission phase. In fact, the magnetic reconnection and the resultant decline of the magnetic flux occurs near the $\mathrm{BH}$ horizon, so that the minimum decay timescale of the extended emission is the light crossing time of the $\mathrm{BH}$ horizon ( $\ll 1 \mathrm{~s})$.

For the light curve, we assume the temporal de- caying index $\alpha=40 / 9$ in Equation (11). This value is calculated from the temporal evolution of the mass accretion rate, $\dot{M} \propto t^{-5 / 3}$ (e.g., Rosswog 2007; Kyutoku et al. 2015). The mass accretion rate from the remnant disk of NS-NS and BH-NS mergers at late phase $(t>1 \mathrm{~s})$ has been studied (e.g., Metzger et al. 2008a, 2010a; Fernández \& Metzger 2013; Metzger \& Fernández 2014; Fernández et al. 2015, 2017). If the effect of the disk outflow is negligible as in the low viscosity case, the mass accretion rate of the advection-dominated disk scales with $\dot{M} \propto t^{-4 / 3}$ (Metzger et al. 2008a). Then, the total fallback mass $M_{\mathrm{f}}$ required for the duration of the plateau emission is about an order of magnitude smaller than the results in Figure 7. On the other hand, the disk winds are powerful and make the time dependence of the accretion rate steepen, $\dot{M} \propto t^{-2.2}$ after $t \geq 1 \mathrm{~s}$ in some simulation results (Fernández et al. 2015, 2017). Then, the required mass $M_{\mathrm{f}}$ becomes large, an order of solar mass $\sim M_{\odot}$ in some fraction of the bursts. In addition, the heating by the decay of $r$-process elements could also affect the accretion rate (Metzger et al. 2010a). Since the current numerical simulations only follow up to $\lesssim 10 \mathrm{~s}$ after the merger (Fernández et al. 2015, 2017) and do not include some important effects such as the magnetic field (e.g., Kiuchi et al. 2015; Siegel \& Metzger 2017), further studies at late phase $(t \gg 10 \mathrm{~s})$ are required.

\section{CONCLUSIONS AND DISCUSSION}

We obtain the statistical properties of the extended and plateau emission components by comparing the phenomenological light curves with the observational data of 65 short GRBs detected by Swift/BAT and XRT. The phenomenological light curve in Equation (11) is found to be consistent with all the observations in our sample. The number of bursts with both the extended and plateau emission components in our sample is 33 , which is more than three times larger than that in previous works (Gompertz et al. 2014; Kisaka \& Ioka 2015). Furthermore, the remaining bursts in our sample (32 bursts) may also have both emission components, which are consistent with the observations (gray dot-dashed curves in Figure 10. This is the first statistical indication that the extended and plateau emission components are ubiquitous in short GRB light curves.

The extended and plateau emission are clearly distinct populations as shown in Figure 4. The ranges of the luminosity and duration for the extended emission are $L_{\text {iso,EX }} \sim 10^{47}-10^{50} \mathrm{erg} \mathrm{s}^{-1}$ and $T_{\mathrm{EX}} \sim 10^{2}-10^{3}$ $\mathrm{s}$, respectively. The ranges extend to the lower and longer values than those of the extended emission detected in the Swift/BAT band (Figure 2). The ranges of the luminosity and duration for the plateau emission, $L_{\text {iso }, \mathrm{PL}} \sim 10^{43}-10^{47} \mathrm{erg} \mathrm{s}^{-1}$ and $T_{\mathrm{PL}} \sim 10^{4}-10^{5} \mathrm{~s}$, respectively, also extend to the lower and longer values than those in the previous works with a small number of sample ( 10; Rowlinson et al. 2013; Gompertz et al. 2014; Kisaka \& Ioka 2015). The isotropic energy of the extended emission $E_{\text {iso,EX }}$ is comparable to that of the prompt emission $E_{\text {iso,PR. }}$. The ratio $E_{\text {iso,EX }} / E_{\text {iso, PR }} \sim$ $0.1-10$ is the same as the short GRBs with the extended emission detected in the Swift/BAT band (e.g., Perley et al. 2009). On the other hand, the isotropic en- 
ergy of the plateau emission, $E_{\text {iso,PL }} \sim 10^{47}-10^{51} \mathrm{erg}$, is $\sim 1-100$ times lower than that of the prompt and the extended emission, $E_{\text {iso,PR }}$ and $E_{\text {iso,EX }}$.

We consider that the extended emission detected by Swift/BAT (e.g., Norris et al. 2011) is the same component detected by Swift/XRT with duration $\sim 10^{2} \mathrm{~s}$. Actually, the luminosity and duration are continuously distributed (Figure 2). The fraction of short GRBs with the extended emission in our sample is $\sim 75 \%$, which is much higher than the previous values, $\sim 2-25 \%$ in the Swift/BAT data (Norris et al. 2010; Sakamoto et al. 2011; Kaneko et al. 2015; Lien et al. 2016), 37.5\% in bright short GRBs detected by both Swift/BAT and XRT (Kagawa et al. 2015), $\sim 5 \%$ in the Fermi/GBM data (Kaneko et al. 2015), and $\sim 7 \%$ in the BATSE data (Bostanci et al. 2013), although these statistical values should not be directly compared because selection criteria are different. The energy spectrum of the extended emission is soft compared with that of the prompt emission (e.g., Kagawa et al. 2015; Lien et al. 2016). In addition, at early phase of the light curve $\sim 10^{2} \mathrm{~s}$, most of our samples are detected by Swift/XRT whose flux sensitivity is much higher than that of Swift/BAT. In fact, the Swift/BAT-detected extended emission has the higher luminosity and shorter duration in the entire extended emission population as shown in Figure 2. These effects would increase the fraction of the XRT-detected bursts with the extended emission. On the other hand, the starting time of the observation by Swift/XRT is usually $60-100 \mathrm{~s}$ after the prompt emission, which is comparable to the duration of the extended emission. Then, Swift/XRT would miss some of the extended emission with short duration, and the actual fraction of the short GRBs with the extended emission could be higher than $\sim 75 \%$.

The fraction of the short GRBs with plateau emission in our samples is also $\sim 75 \%$. Since the plateau emission is generally $\sim 10^{-3}-10^{-4}$ times dimmer than the extended emission (Figure 3 A), the flux of the plateau emission in some bursts would be below the detection limit. Actually, the upper limit on the luminosity of the plateau emission in our sample is typically $\sim 10^{44}-10^{45}$ erg s$^{-1}$ (gray dot-dashed curves in Figure 1), higher than the lower end of the luminosity distribution of plateau emission (Figure 2) at the time $\sim 10^{5}$ s. Therefore, the actual fraction would be higher than $\sim 75 \%$, and it is consistent with that almost all short GRBs have the plateau emission component.

There is a possible indication for the short- and longlived populations of the light curves at the plateau phase, $t>10^{4} \mathrm{~s}$ (Sakamoto \& Gehrels 2009). From Figure 2(B), the distribution may be composed by two distinct populations, $T_{\mathrm{PL}} \sim 10^{4} \mathrm{~s}$ and $T_{\mathrm{PL}} \sim 10^{5} \mathrm{~s}$, although it is not so obvious in our sample.

The X-ray excess component with timescale $\sim 10^{6}$ $\mathrm{s}$, longer than the plateau emission, has also detected by XMM-Newton and Chandra in some bursts (e.g., Fong et al. 2014, 2016). For the excess component, the activity near the central engine such as an accretion disk is considered (e.g., Rosswog 2007; Rossi \& Begelman 2009; Kyutoku et al. 2015; Kisaka et al. 2016). For the excess component detected by $X M M-N$ ewton in GRB 130603B, the fallback mass $\sim 0.02 M_{\odot}$ are required to produce the observed luminosity $\sim 10^{41} \mathrm{erg} \mathrm{s}^{-1}$ at $\sim 7$ day in the rest-frame if the radiation efficiency of the accretion disk is $\sim 0.1$ and the mass accretion rate follows $\dot{M} \propto t^{-5 / 3}$ at $t>0.1 \mathrm{~s}$ (Kisaka et al. 2016). This is consistent with the mass $M_{\mathrm{f}}$ from the observed plateau emission parameters if the radiation efficiency of the jet $\eta \sim 2.5 \times 10^{-2}$ at the plateau emission phase is assumed. From Figure 7, a fraction 17\% of bursts with the plateau emission requires the fallback mass $M_{\mathrm{f}} \gtrsim 0.02 M_{\odot}$, so that these bursts could have the X-ray excess with $L \gtrsim 10^{41} \mathrm{erg} \mathrm{s}^{-1}$ at $\sim 7$ day in the rest-frame. The excess component could also contribute to the energy source of the observed macronovae Kisaka et al. 2016; Jin et al. 2016).

We use the light curve data from UK Swift Science Data Center (Evans et al. 2007, 2009). In some data, the exposure time is much shorter than the error duration. We show the data points with the low fractional exposure $(<0.1)$ as gray points in Figure1. Even if we do not use such data, the results do not significantly change as shown in Figure 11. For the last data point in some GRBs, even if the fractional exposure is close to unity, the arrival time of almost all the photons in the bin is clustering in the first short time range compared with the error duration. Then, we may overestimate the duration in the light curve fitting. A example is the last data point in GRB 090515, which is divided by the detection point with duration $<10^{2} \mathrm{~s}$ and the upper limit with $\sim 10^{3} \mathrm{~s}$ in the analysis by Rowlinson et al. (2010a). Note that for GRB 090515, the last data point corresponds to the decay phase of the extended emission (Figure 11), which could decay more rapidly than the model light curve. We consider that such data points do not significantly change our results.

The emission solid angles of the extended and plateau emission are unknown. If the emission is isotropic, the extended and plateau emission could be easily detectable as EM counterparts to the NS binary mergers. For the Swift/BAT-detected extended emission, Nakamura et al. (2014) estimated the emission solid angle, $\sim 10^{-3}$ steradian, by comparing the detection rate by Swift/BAT with the estimated merger rate $\sim 10^{3} \mathrm{Gpc}^{-3}$ $\mathrm{yr}^{-1}$.

From our results, the extended emission is mainly detected in the Swift/XRT band, and the luminosity range is $10^{47} \mathrm{erg} \mathrm{s}^{-1} \lesssim L_{\text {iso,EE }} \lesssim 10^{50} \mathrm{erg} \mathrm{s}^{-1}$. The all-sky survey in soft $\mathrm{X}$-ray band is planned by eROSITA. Let us consider the detection rate of the extended emission by $e R O S I T A$ as a function of the emission solid angle, $\Delta \Omega_{\mathrm{EX}}$. The flux sensitivity limit of the eROSITA single survey pass is $\sim 10^{-13} \mathrm{erg} \mathrm{cm}^{-2} \mathrm{~s}^{-1}$ (Merloni et al. 2012), which corresponds to the detection horizon $\sim 100$ $\mathrm{Gpc}$ in the luminosity distance and the comoving volume $4 \times 10^{3} \mathrm{Gpc}^{3}$ for the luminosity $\sim 10^{47} \mathrm{erg} \mathrm{s}^{-1}$. Then, the event rate in the volume is $\sim 0.1 \mathrm{~s}^{-1}$ for the NS binary merger rate $R_{\text {merger }} \sim 10^{3} \mathrm{Gpc}^{-3} \mathrm{yr}^{-1}$. Using the typical duration $\sim 100 \mathrm{~s}$, there are always $\sim 10$ events in the all-sky. Since a field-of-view of eROSITA is 0.833 $\mathrm{deg}^{2}$ and the scanning speed is one full circle per four hours (Merloni et al. 2012), the detection rate is relatively high $\sim 0.5\left(\Delta \Omega_{\mathrm{EX}} / 4 \pi\right)\left(R_{\text {merger }} / 10^{3} \mathrm{Gpc}^{-3} \mathrm{yr}^{-1}\right)$ day $^{-1}$. The events will be detected only in one scanning, in contrast to stationary sources. Therefore, eROSITA 
could significantly constrain the emission solid angle of the extended emission.

If the extended emission is isotropic, off-axis events should appear as short GRB-less X-ray flashes or long GRBs with relatively simple shaped light curves and unusual host galaxy properties compared to normal long GRBs. XRF GRB060428b, which had a light curve similar to an observed X-ray flash and which was localized to a potential host elliptical galaxy (Perley et al. 2007), was suggested as a possible off-axis extended emission of short GRB (Metzger et al. 2008b). We will investigate long GRB afterglows with similar light curves to short GRBs in future work.

In the light curves of long GRBs, the existence of a single long-lasting component, so-called the plateau emission in addition to the prompt emission and $\mathrm{X}$ ray flares, has been established (e.g., O'Brien et al. 2006; Willingale et al. 2007; Ghisellini et al. 2009; Yamazaki 2009; Grupe et al. 2013; Rowlinson et al. 2014; Dainotti et al. 2015; Wang et al. 2015). The duration distributions of the extended and plateau emission in short GRBs (Figure 3 B) are similar to those of the prompt and plateau emission in long GRBs, respectively (e.g., Willingale et al. 2007). In addition, the distribution of the fluence ratio between the extended and plateau emission in short GRBs (Figure 5 A) is also similar to that of the ratio between the prompt and plateau emission in long GRBs (e.g., Willingale et al. 2007). These similarities may suggest that the physical conditions of the model of Kisaka \& Ioka (2015) are realized in both short and long GRBs. In fact, the central engine activities, mass ejection, and fallback accompanied by supernova explosion are also expected in long GRBs.

There are some caveats to be addressed in the future work. First, we fit the phenomenological light curve with the observations by eye inspection, because the light curves often have additional complex structures of Xray flares (e.g., Margutti et al. 2011). The observed data of most bursts would be insufficient to separate such complex structures. In order to separate the additional structures from the flat component, a sample with more sufficient X-ray data is required.

Second, we also neglect the contribution of the normal afterglow in the X-ray light curve observed by Swift. Although the normal afterglow may contribute to some bursts whose light curves are consistent with a single power-law form (e.g., Lü et al. 2015), these light curves are also able to fit with the extended and plateau emissions as demonstrated in this paper. The isotropic energies of the extended and plateau emission components are almost comparable to that of the prompt emission, $E_{\text {iso, } \mathrm{EX}} / E_{\text {iso, } \mathrm{PR}} \sim 0.1-10$ and $E_{\text {iso, } \mathrm{PL}} / E_{\text {iso, } \mathrm{PR}} \sim 0.01-1$, as shown in Figure 5, which would also suggest the continuous energy injection from the central engine. Note that most of the Swift/XRT data are within $\lesssim 10^{5}$ s after the prompt emission, so that the normal afterglow would significantly contribute to the X-ray light curve at later time $\left(\geq 10^{5} \mathrm{~s}\right)$, at which the jet break is seen (e.g., Fong et al. 2015).

Third, we use the specific value $\alpha=40 / 9$ for the phenomenological light curve. Although the parameters $L_{\text {iso,EX }}, L_{\text {iso,PL }}, T_{\mathrm{EX}}$, and $T_{\mathrm{PL}}$ do not significantly depend on $\alpha$ (see the dotted lines $(\alpha=5 / 3)$ in Figure 1 ), the light curve with small $\alpha$ could explain the observed data using a single emission component in a few bursts. Then, the fraction of bursts with the plateau emission would slightly decrease. On the other hand, the light curve model with small $\alpha$ is not consistent with some bursts with the rapidly decaying plateau emission. In the theoretical point of view, the parameter $\alpha$ depends on the accretion model as discussed in Section 5. A sample with sufficient X-ray data will provide the fitted value of $\alpha$ to characterize the light curve and a clue to the fallback to the central engine.

Recently, a new X-ray transient detected by Chandra has been reported (Bauer et al. 2017). Using the measured redshift $z \sim 2.2$ (Bauer et al. 2017), the luminosity $\sim 10^{47} \mathrm{erg} \mathrm{s}^{-1}$ and duration $\sim 10^{2} \mathrm{~s}$ could be consistent with the extended emission properties. The decaying light curve of the X-ray transient seems to become gradually shallow (in their Figure 4), so that the plateau emission may also contribute to the observed light curves. For the prompt emission properties, the interplanetary network (IPN; Atteia et al. 1987) gives the limits on the fluence and peak photon flux $\left(<10^{-6}\right.$ erg $\mathrm{cm}^{-1}$ and $<1$ photon $\mathrm{cm}^{-2} \mathrm{~s}^{-1}$, respectively, in the 25-150 keV range; Bauer et al. 2017). A fraction of the prompt emission of short GRBs is fainter than the limits (Lien et al. 2016). Within the $2 \sigma$ range of the measured photometric redshift, the host galaxy could locate at $z \sim 0.39$ (Bauer et al. 2017). If we use this value, the luminosity $\sim 10^{44} \mathrm{erg} \mathrm{s}^{-1}$ and duration $\sim 10^{3} \mathrm{~s}$ are consistent with those of the scattered plateau emission. In addition, the event rate of the transient is $\sim 10^{2}-10^{3}$ $\mathrm{yr}^{-1} \mathrm{Gpc}^{-3}$ in the low $z(\lesssim 1)$ case, which is also consistent with the rate of the orphan short GRBs (Berger 2014; Bauer et al. 2017).

Possible macronova emission was reported in short GRB 160821B (Tanvir et al. 2017; Troja et al. 2016b; Kasliwal et al. 2017), which occurred at $z \sim 0.16$ (Levan et al. 2016), closer than GRB 130603B. The peak luminosity is comparable to that of GRB 130603B while the peak time is earlier, $t \sim 3$ day. The observed luminosity is consistent with the expected bolometric luminosity, $\sim 10^{41}(t / 3 \text { day })^{-2} \mathrm{erg} \mathrm{s}^{-1}$, in the case of ejecta heated by the plateau activity (Kisaka et al. 2015b), where we use $\eta=0.1, \theta_{\mathrm{j}}=0.1$, and the observed value $L_{\text {iso,PL }} T_{\mathrm{PL}}^{2}$ in GRB $160821 \mathrm{~B}$, which is $\sim 30$ times smaller than that of GRB 130603B. Note that the possible X-ray excess component $\left(\lesssim 10^{42} \mathrm{erg} \mathrm{s}^{-1}\right.$ at $10^{6} \mathrm{~s}$ by Swift/XRT, see Figure 1) could also contribute to heating the ejecta (Kisaka et al. 2016). Therefore, the enginepowered macronova scenario is consistent with the observations so far.

We are grateful to the anonymous referee for constructive comments. We would like to thank Amy Lien for kindly providing the data of the short pulse fluence of short GRBs with extended emission, and $\mathrm{Yu}-$ taka Ohira, Masaomi Tanaka, and Ryo Yamazaki for fruitful discussions. This work is supported by KAKENHI 16J06773 (S.K.), 24103006, 26247042, 26287051, 17H01126, 17H06131, $17 \mathrm{H} 06357$ (K.I.), and $17 \mathrm{H} 06362$ (K.I., T.S.). 


\section{REFERENCES}

Abbott, B. P., Abbott, R., Abbott, T. D., et al. 2016a, PhRvL, 116,061102

Abbott, B. P., Abbott, R., Abbott, T. D., et al. 2016b, PhRvL, 116,241103

Abbott, B. P., Abbott, R., Abbott, T. D., et al. 2016c, PhRvX, 6, 041015

Abbott, B. P., Abbott, R., Abbott, T. D., et al. 2016d, ApJL, 832, L21

Abbott, B. P., Abbott, R., Abbott, T. D., et al. 2017, PhRvL, 118,221101

Adriani, O., Akaike, Y., Asano, K., et al. 2016, ApJL, 829, L20

Atteia, J.-L., Barat, C., Hurley, K., et al. 1987, ApJS, 64, 305

Barkov, M. V., \& Pozanenko, A. S. 2011, MNRAS, 417, 2161

Barthelmy, S. D., Cannizzo, J. K., Gehrels, N., et al. 2005, ApJL, 635, L133

Bauer, F. E., Treister, E., Schawinski, K., et al. 2017, MNRAS, 467,4841

Berger, E. 2006, GCN, 5952, 1

Berger, E. 2009, ApJ, 690, 231

Berger, E. 2014, ARA\&A, 52, 43

Berger, E., Fong, W., \& Chornock, R. 2013a, ApJL, 774, L23

Berger, E., Fox, D. B., Price, P. A., et al. 2007, ApJ, 664, 1000

Berger, E., Price, P. A., Cenko, S. B., et al. 2005, Natur, 438, 988

Berger, E., Zauderer, B. A., Levan, A., et al. 2013b, ApJ, 765, 121

Blandford, R. D., \& Znajek, R. L. 1977, MNRAS, 179, 433

Bostancı, Z. F., Kaneko, Y., \& Göğüş, E. 2013, MNARS, 428, 1623

Bromberg, O., Nakar, E., Piran, T., \& Sari, R. 2011, ApJ, 740, 100

Bucciantini, N., Metzger, B. D., Thompson, T. A., \& Quataert, E. 2012, MNRAS, 419, 1537

Camp, J., Barthelmy, S. D., Blackburn, L., et al. 2013, ExA, 36, 505

Castro-Tirado, A. J., Sanchez-Ramirez, R., Lombardi, G., et al. 2015, GCN, 17758, 1

Cenko, S. B., Berger, E., Nakar, E., et al. 2008, arXiv:0802.0874

Chen, H.-Y., \& Holz, D. E. 2015, arXiv:1509.00055

Chornock, R., \& Fong, W. 2015, GCN, 17358, 1

Chornock, R., Lunnan, R., \& Berger, E. 2013, GCN, 15307, 1

Cucchiara, A., \& Levan, A. J. 2016, GCN, 19565, 1

D'Avanzo, P., Malesani, D., Covino, S., et al. 2009, A\&A, 498, 711

D'Avanzo, P., Salvaterra, R., Bernardini, M. G., et al. 2014, MNRAS, 442, 2342

Dainotti, M., Petrosian, V., Willngale, R., et al. 2015, MNRAS, 451,3898

de Ugarte Postigo, A., Thöne, C. C., Rowlinson, A., et al. 2014, A\&A, 563, A62

Della Valle, M., Chincarini, G., Panagia, N., et al. 2006, Natur, 444, 1050

Evans, P. A., Beardmore, A. P., Page, K. L., et al. 2007, A\&A, 469, 379

Evans, P. A., Beardmore, A. P., Page, K. L., et al. 2009, MNRAS, 397,1177

Fan, X., Messenger, C., \& Heng, I. S. 2017, arXiv:1706.05639

Fernández, R., \& Metzger, B. D. 2013, MNRAS, 435, 502

Fernández, R., Quataert, E., Schwab, J., Kasen, D., \& Rosswog, S. 2015, MNRAS, 449, 390

Fernández, R., Foucart, F., Kasen, D., et al. 2017, CQG, 34, 154001

Fong, W., Berger, E., Chornock, R., et al. 2013, ApJ, 769, 56

Fong, W., Berger, E., Chornock, R., et al. 2011, ApJ, 730, 26

Fong, W., Berger, E., Margutti, R., \& Zauderer, B. A. 2015, ApJ, 815,102

Fong, W., Berger, E., Metzger, B. D., et al. 2014, ApJ, 780, 118

Fong, W., Margutti, R., Chornock, R., et al. 2016, ApJ, 833, 151

Foucart, F., Desai, D., Brege, W., et al. 2017, CQG, 34, 044002

Foucart, F., O'Connor, E., Roberts, L., et al. 2015, PhRvD, 91, 124021

Fraley, C., \& Raftery, A. E. 2002, JASA, 97, 611

Gao, W.-H., \& Fan, Y.-Z. 2006, ChJAA, 6, 513

Gehrels, N., Norris, J. P., Barthelmy, S. D., et al. 2006, Natur, 444, 1044

Ghisellini, G., Nardini, M., Ghirlanda, G., \& Celotti, A. 2009, MNRAS, 393, 253
Gibson, S. L., Wynn, G. A., Gompertz, B. P., \& O'Brien, P. T. 2017, arXiv:1706.04802

Gompertz, B. P., O’Brien, P. T., \& Wynn, G. A. 2014, MNRAS, 438,240

Gompertz, B. P., O’Brien, P. T., Wynn, G. A., \& Rowlinson, A. 2013, MNRAS, 431, 1745

Gottlieb, O., Nakar, E., \& Piran, T. 2017, arXiv:1705.10797

Grupe, D., Nousek, J. A., Veres, P., Zhang, B.-B., \& Gehrels, N. 2013, ApJS, 209, 20

Hartigan, P. M., 1985, Appl. Stat., 34, 320

Hotokezaka, K., Kiuchi, K., Kyutoku, K., et al. 2013, PhRvD, 87, 024001

Hotokezaka, K., \& Piran, T. 2015, MNRAS, 450, 1430

Ioka, K., Kobayashi, S., \& Zhang, B. 2005, ApJ, 631, 429

Jin, Z.-P., Hotokezaka, K., Li, X., et al. 2016, NatCo, 7, 12898

Kagawa, Y., Yonetoku, D., Sawano, T., Toyanago, A., Nakamura, T., Takahashi, K., Kashiyama, K., \& Ioka, K. 2015, ApJ, 811, 4

Kaneko, Y., Bostancı, Z. F., Göğüs, E., \& Lin, L. 2015, MNARS, 452,824

Kanner, J., Camp, J., Racusin, J., Gehrels, N., \& White, D. 2012, ApJ, 759, 22

Kasen, D., Badnell, N. R., \& Barnes, J. 2013, ApJ, 774, 25

Kasliwal, M. M., Korobkin, O., Lau, R. M., Wollaeger, R., \& Fryer, C. L. 2017, ApJL, 843, L34

Kawaguchi, K., Kyutoku, K., Nakano, H., Okawa, H., Shibata,

M., \& Taniguchi, K. 2015, PhRvD, 92, 024014

Kisaka, S., \& Ioka, K. 2015, ApJL, 804, L16

Kisaka, S., Ioka, K., \& Nakamura, T. 2015b, ApJL, 809, L8

Kisaka, S., Ioka, K., \& Nakar, E. 2016, ApJ, 818, 104

Kisaka, S., Ioka, K., \& Takami, H. 2015a, ApJ, 802, 119

Kiuchi, K., Sekiguchi, Y., Kyutoku, K., Shibata, M., Taniguchi, K., \& Wada, T. 2015, PhRvD, 92, 064034

Kouveliotou, C., Meegan, C. A., Fishman, G. J., et al. 1993, ApJL, 413, L101

Krimm, H. A., Holland, S. T., Corbet, R. H. D., et al. 2013, ApJS, 209, 14

Kulkarni, S. R. 2005, astro-ph/0510256

Kyutoku, K., Ioka, K., Okawa, H., Shibata, M., \& Taniguchi, K. 2015, PhRvD, 92, 044028

Lazzati, D., Deich, A., Morsony, B. J., \& Workman, J. C. 2016, arXiv: 1610.01157

Lee, W. H., \& Ramirez-Ruiz, E. 2007, NJPh, 9, 17

Leibler, C. N., \& Berger, E. 2010, ApJ, 725, 1202

Levan, A. J., Wiersema, K., Tanvir, N. R., et al. 2016, GCN, 19846, 1

Levesque, E. M., Bloom, J. S., Butler, N. R., et al. 2010, MNRAS, 401, 963

Li, L.-X., \& Paczyńsky, B. 1998, ApJL, 507, L59

Lien, A., Sakamoto, T., Barthelmy, S. D., et al. 2016, ApJ, 829, 7

Lü, H.-J., Zhang, B., Lei, W.-H., Li, Y., \& Lasky, P. D. 2015, ApJ, 805, 89

Lü, H.-J., Zhang, H.-M., Zhong, S.-Q., et al. 2017, ApJ, 835, 181

Lyne, A. G., Burgay, M., Kramer, M., et al. 2004, Sci, 303, 1153

Lyutikov, M. 2013, ApJ, 768, 63

Malesani, D., Kruehler, T., Xu, D., et al. 2015, GCN, 17755, 1

Margutti, R., Chincarini, G., Granot, J., et al. 2011, MNRAS, 417,2144

McBreen, S., Krühler, T., Rau, A., et al. 2010, A\&A, 516, A71

Merloni, A., Predehl, P., Becker, W., et al. 2012, arXiv:1209.3114

Metzger, B. D., Arcones, A., Quataert, E., \& Martínez-Pinedo, G. 2010a, MNRAS, 402, 2771

Metzger, B. D., \& Berger, E. 2012, ApJ, 746, 48

Metzger, B. D., \& Fernández, R. 2014, MNRAS, 441, 3444

Metzger, B. D., Martínez-Pinedo, G., Darbha, S., Quataert, E., Arcones, A., Kasen, D., Thomas, R., Nugent, P., Panov, I. V., \& Zinner, N. T. 2010b, MNRAS, 406, 2650

Metzger, B. D. \& Piro, A. L. 2014, MNRAS, 439, 3916

Metzger, B. D., Piro, A. L., \& Quataert, E. 2008a, MNRAS, 390, 781

Metzger, B. D., Quataert, E., \& Thompson, T. A. 2008b, MNRAS, 385, 1455

Nagakura, H., Hotokezaka, K., Sekiguchi, Y., Shibata, M., \& Ioka, K. 2014, ApJL, 784, L28

Nakamura, T., Kashiyama, K., Nakauchi, D., et al. 2014, ApJ, 796,13 
Nakar, E. 2007, PhR, 442, 166

Narayan, R., Igumenshchev, I. V., \& Abramowicz, M. A. 2003, PASJ, 55, L69

Narayan, R., Paczyńsky, B., \& Piran, T. 1992, ApJL, 395, L83

Narayana Bhat, P., Meegan, C. A., von Kienlin, A., et al. 2016, ApJS, 223, 28

Norris, J. P., \& Bonnell, J. T. 2006, ApJ, 643, 266

Norris, J. P., Gehrels, N., \& Scargle, J. D. 2011, ApJ, 735, 23

Norris, J. P., Gehrels, N., \& Scargle, J. D. 2010, ApJ, 717, 411

O'Brien, P. T., Willingale, R., Osborne, J., et al. 2006, ApJ, 647, 1213

Perley, D. A., Bloom, J. S., Butler, N. R., Li, W., \& Chen, H.-W. 2007, in AIP Conf. Proc. 937, Supernova 1987A: 20 Years After: Supernovae and Gamma-Ray Bursters, ed. S. Immler, K. Weiler, \& R. McCray, 526

Perley, D. A., Bloom, J. S., Modjaz, M., et al. 2008, GCN, 7889, 1

Perley, D. A., Metzger, B. D., Granot, J., et al. 2009, ApJ, 696, 1871

Prochaska, J. X., Bloom, J. S., Chen, H.-W., et al. 2005, GCN, 3399, 1

Rossi, E. M., \& Begelman, M. C. 2009, MNRAS, 392, 1451

Rosswog, S. 2007, MNRAS, 376, L48

Rowlinson, A., Gompertz, B. P., Dainotti, M., et al. 2014, MNRAS, 443, 1779

Rowlinson, A., O'Brien, P. T., Metzger, B. D., Tanvir, N. R., \& Levan, A. J. 2013, MNRAS, 430, 1061

Rowlinson, A., O'Brien, P. T., Tanvir, N. R., et al. 2010a, MNRAS, 409, 531

Rowlinson, A., Wiersema, K., Levan, A. J., et al. 2010b, MNRAS, 408,383

Sakamoto, T., Barthelmy, S. D., Baumgartner, W. H., et al. 2011, ApJS, 195, 27

Sakamoto, T., \& Gehrels, N. 2009, in AIP Conf. Proc. 1133, Gamma-Ray Bursts, 6th Huntsville Symposium, ed. C.

Meegan, N. Gehrels, \& C. Kouveliotou (Melville, NY: AIP), 112

Sakamoto, T., Troja, E., Aoki, K., et al. 2013, ApJ, 766, 41
Sari, R., Piran, T., \& Narayan, R. 1998, ApJL, 497, L17

Savchenko, V., Ferrigno, C., Mereghetti, S., et al. 2016, ApJL, 820 , L36

Shibata, M., \& Taniguchi, K. 2006, PhRvD, 73, 064027

Siegel, D. M., \& Metzger, B. D. 2017, arXiv:1705.05473

Singer, L. P., \& Price, L. R. 2016, PhRvD, 93, 024013

Singer, L. P., Price, L. R., Farr, B., et al. 2014, ApJ, 795, 105

Soderberg, A. M., Berger, E., Kasliwal, M., et al. 2006, ApJ, 650, 261

Sugizaki, M., Mihara, T., Serino, M., et al. 2011, PASJ, 63, 635

Sun, H., Zhang, B., \& Gao, H. 2017, ApJ, 835, 7

Tanaka, M., \& Hotokezaka, K. 2013, ApJ, 775, 113

Tanvir, N. R., et al. 2017, The Physics of Extreme-Gravity Stars, Stockholm, Sweden

Tanvir, N. R., Levan, A. J., Fruchter, A. S., et al. 2013, Natur, 500,547

Tchekhovskoy, A., \& Giannios, D. 2015, MNRAS, 447, 327

Tchekhovskoy, A., Narayan, R., \& McKinney, J. C. 2011,

MNRAS, 418, L79

Thoene, C. C., de Ugarte Postigo, A., Vreeswijk, P. 2010, GCN, 10971, 1

Troja, E., Sakamoto, T., Cenko, S. B., et al. 2016a, ApJ, 827, 102

Troja, E., Tanvir, N., Cenko, S. B., et al. 2016b, GCN, 20222, 1

Usov, V. V. 1992, Natur, 357, 472

Wang, X.-G., Zhang, B., Liang, E.-W., et al. 2015, ApJS, 219, 9

Willingale, R., O’Brien, P. T., Osborne, J. P., 2007, ApJ, 662, 1093

Yamazaki, R. 2009, ApJL, 690, L118

Yu, Y.-W., Zhang, B., \& Gao, H. 2013, ApJL, 776, L40

Yuan, W., Zhang, C., Feng, H., et al. 2015, arXiv:1506.07735

Zamaninasab, M., Clausen-Brown, E., Savolainen, T., \& Tchekhovskoy, A. 2014, Natur, 510, 126

Zhang, B., Liang, E., Page, K. L., et al. 2007, ApJ, 655, 989

Zhang, B., \& Mészáros, P. 2001, ApJL, 552, L35 